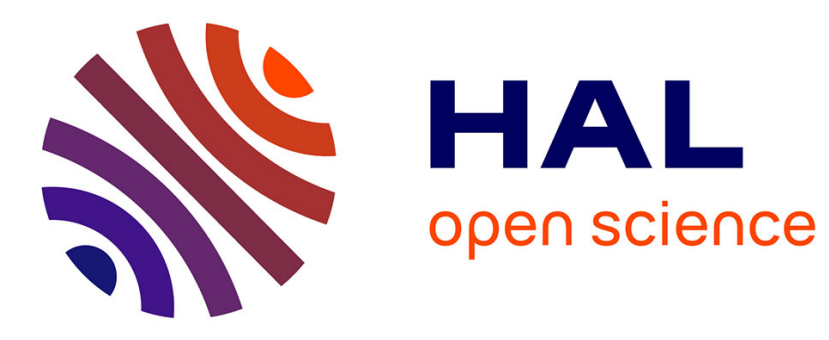

\title{
Event Structure Semantics of Orc
}

Sidney Rosario, David Kitchin, Albert Benveniste, William J. Cook, Stefan Haar, Claude Jard

\section{To cite this version:}

Sidney Rosario, David Kitchin, Albert Benveniste, William J. Cook, Stefan Haar, et al.. Event Structure Semantics of Orc. [Research Report] PI 1853, 2007, pp.33. inria-00155782

\section{HAL Id: inria-00155782 https://hal.inria.fr/inria-00155782}

Submitted on 19 Jun 2007

HAL is a multi-disciplinary open access archive for the deposit and dissemination of scientific research documents, whether they are published or not. The documents may come from teaching and research institutions in France or abroad, or from public or private research centers.
L'archive ouverte pluridisciplinaire HAL, est destinée au dépôt et à la diffusion de documents scientifiques de niveau recherche, publiés ou non, émanant des établissements d'enseignement et de recherche français ou étrangers, des laboratoires publics ou privés. 


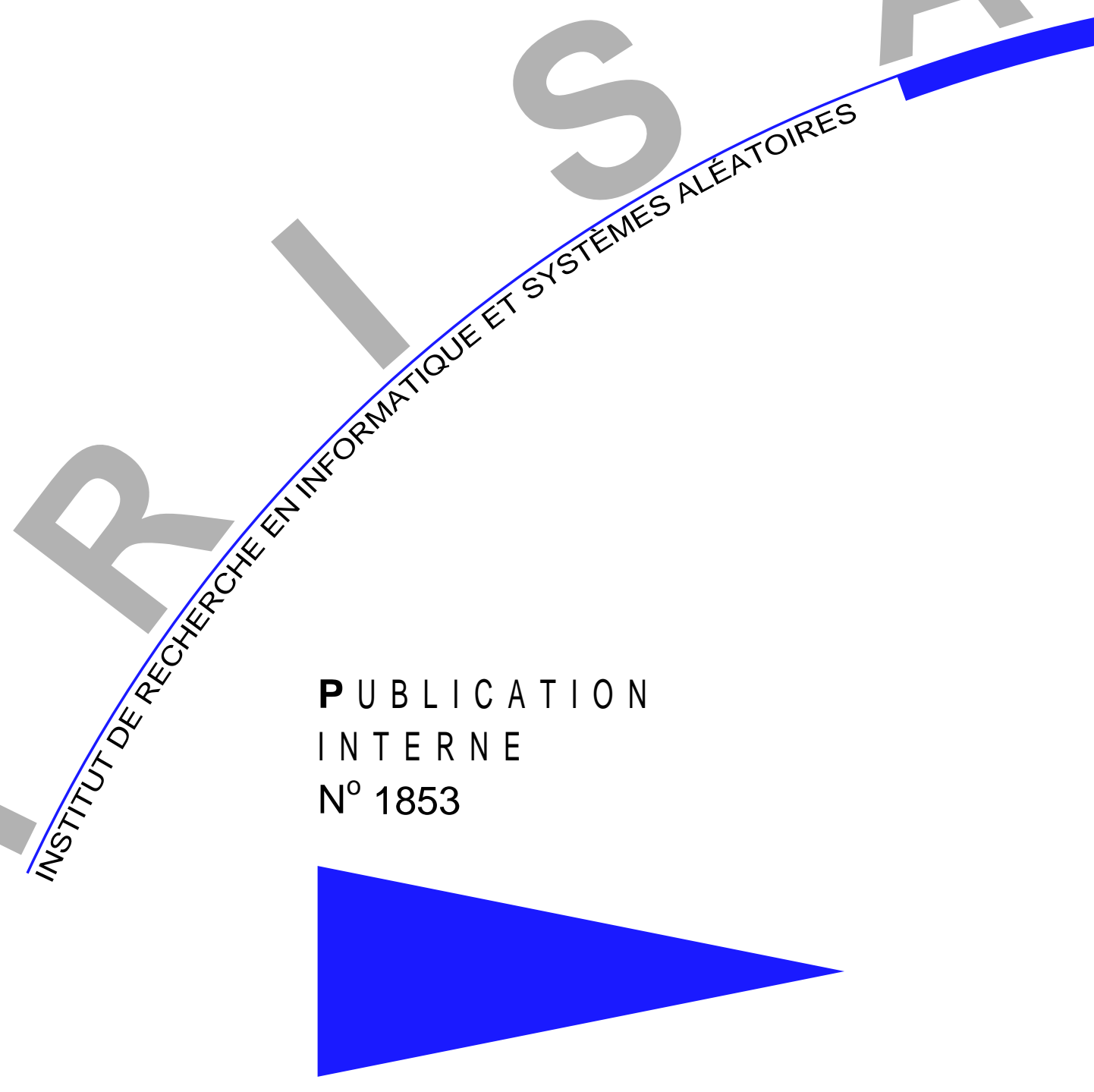

EVENT STRUCTURE SEMANTICS OF ORC

SIDNEY ROSARIO , DAVID KITCHIN , ALBERT BENVENISTE, WILLIAM COOK, STEFAN HAAR, CLAUDE JARD 



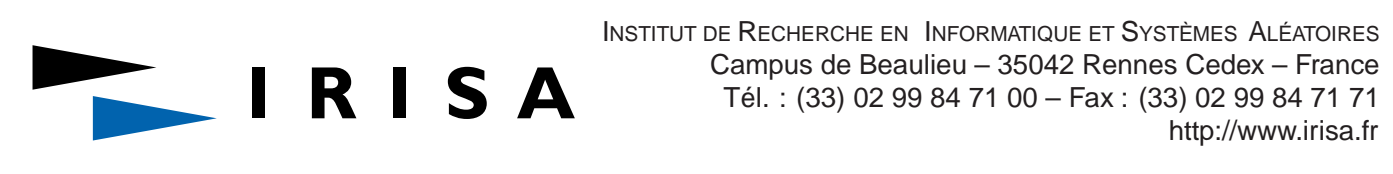

\title{
Event Structure Semantics of Orc ${ }^{*}$
}

\author{
Sidney Rosario, David Kitchin, Albert Benveniste, William Cook, Stefan \\ Haar , Claude Jard \\ Systèmes communicants \\ Projets Distribcom \\ Publication interne $\mathrm{n}^{\circ} 1853$ - Juin 2007 - 33 pages
}

\begin{abstract}
One challenge in developing wide-area distributed applications is analyzing the system's non-functional properties, including timing constraints and internal dependencies that can affect quality of service. Analysis of non-functional properties requires a precise formal semantics for the language in which the system is written; but labelled transition systems and trace semantics, which are commonly used for this purpose, do not facilitate this kind of analysis. Event structures provide an explicit representation of the the causal dependencies between events in the execution of a system. But event structures are difficult to construct compositionally, because they cannot easily represent fragments of a computation. In this paper we present a partial-order semantics based on heaps (an explicitly encoded form of occurrence nets with read arcs), which naturally represent fragments of behavior. Heaps are then easily translated into asymmetric event structures. The semantics is developed for Orc, an orchestration language in which concurrent services are invoked to achieve a goal while managing time-outs, exceptions, and priority. Orc, and this new semantics, are being used to study quality of service (QoS) for wide area orchestrations.
\end{abstract}

Key-words: partial orders, event structures, semantics

(Résumé : tsvp)

\footnotetext{
* This work has been supported in part by the following projects: French national telecommunication research network (RNRT) project SWAN, decision no. 03 S 481, and by ANR-06-MDCA-005 Project DocFlow.

** S. Rosario, A. Benveniste, S. Haar are with Irisa/Inria, Campus de Beaulieu, Rennes. D. Kitchin and W. Cook are with the Department of Computer Science, University of Texas, Austin. C. Jard is with Irisa/ENS Cachan Campus de Beaulieu, Rennes.
}

\section{coss}

Centre National de la Recherche Scientifique (UMR 6074) Université de Rennes 1 - Insa de Rennes
Institut National de Recherche en Informatique et en Automatique - unité de recherche de Rennes 


\section{Semantique en structures d'eventments pour Orc}

Résumé : Dans cet article nous proposons une sémantique dénotationelle en terms de structures d'événements pour le langage Orc. Orc est un langage pour modéliser des orchestrations sur l'internet. On utilise Orc et ce nouveau sémantique pour étudier les aspects de 'qualité de service' pour des orchestrations.

Mots clés : ordre partiels, structures d'événements, sémantique 


\section{Contents}

\begin{tabular}{|lc}
\hline Introduction & 4
\end{tabular}

2 Asymmetric Event Structures and Heaps 5

2.1 Asvmmetric Event Structures with Labels . . . . . . . . . . . . . . . . . . 5

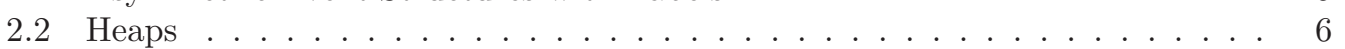

2.3 From Heaps to LAES . . . . . . . . . . . . . . . . . . . . . . . . . . . . . . 9

2.4 Generic Operations on Heaps . . . . . . . . . . . . . . . . . . . . . . . 9

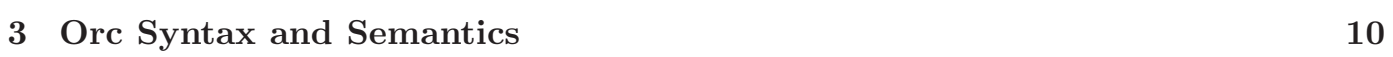

4 Denotations for Orc Expressions $\quad 11$

4.1 Heaps of Base Expressions . . . . . . . . . . . . . . . . . . . . . . . 13

4.2 Heans for the Combinators . . . . . . . . . . . . . . . . . . . . . . . . . . . . . . . . . 13

4.3 Recursive Definitions . . . . . . . . . . . . . . . . . . . . . . . . 14

5 Correctness of Orc heap semantics $\quad 16$

$\begin{array}{llr}6 & \text { Related Work } & 17\end{array}$

\begin{tabular}{|lr}
\hline 7 Conclusion & 17
\end{tabular}

$\begin{array}{lr}\text { A Proof of Theorem居 } & 20\end{array}$

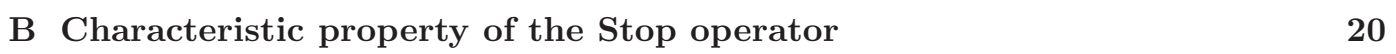

$\begin{array}{lr}\text { C Proof of Lemma 2 } & 21\end{array}$

D Proof of Correspondence Theorem $\mathbf{2} \quad 22$

D.1 Definition of Terms . . . . . . . . . . . . . . . . . . . . . . 22

D.1.1 Linear extension of a trace . . . . . . . . . . . . . . . . . . . . . . . . . . . . . 22

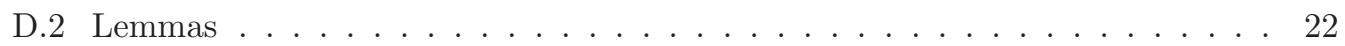

D.3 Correspondence Theorem . . . . . . . . . . . . . . . . . . 25

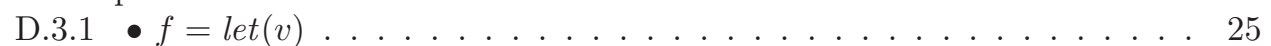

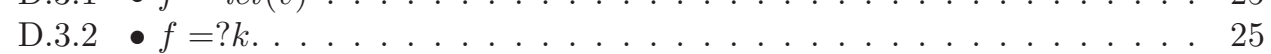

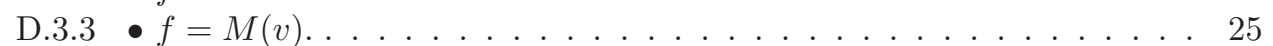

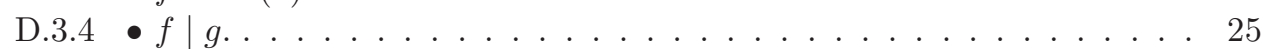

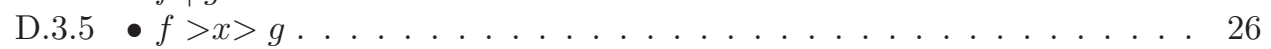

D.3.6 $\bullet f$ where $x: \in q$. . . . . . . . . . . . . . . . . . 29 


\section{Introduction}

Orc is a structured language for computation orchestration, in which concurrent services are invoked to achieve a goal while managing time-outs, exceptions, and priority 12 . The operational semantics of Orc was first defined as a labeled transition system. A denotational semantics of Orc has also been defined; the denotations are sets of traces, which explicitly represent the observable behavior of an Orc program [7]. For other studies of Orc semantics see [3], where the authors link the Orc language to Petri nets and the join calculus, and [14, where Orc expressions are translated to colored Petri net systems. On the other hand, a number of papers have been devoted to the semantics of the most widely used language for orchestration, namely BPEL, see [6, 9, 13, 15, 8] and the tutorial [16]. Still, very little has been done toward getting, for orchestration languages, a semantics that is suitable for Quality of Service (QoS) studies.

Analyzing QoS or non-functional properties, like timing constraints derived from the critical path of dependencies, can be quite difficult with either an operational or a denotational trace semantics. The problem is that neither of these semantics exhibits the causality constraints that govern concurrent execution. These causality constraints can be represented explicitly as partial orders over events. With a partial order semantics, analysis and verification of programs are facilitated, and translations between different formalisms can be checked for correctness. Last but not least, partial order representations are crucial for evaluating overall durations of programs: time-consuming actions that run in parallel increase the overall delay less than actions that have to occur sequentially; see [11, 10] for more on this type of dynamics. The partial order semantics is therefore crucial for the QoS analyses for orchestrated services [14. In this paper we develop a partial order semantics of Orc in terms of asymmetric event structures [1]. An event structure is a set of events with one or more relations that constrain the allowed sequences of events. Asymmetric event structures have an asymmetric conflict relation, $a \nearrow b$, which states that event $b$ cannot precede event $a$ in a same execution. Asymmetric conflict is convenient to express preemption or termination, which is an essential feature needed for wide area computing and offered by Orc. In Orc, an execution $A$ can be preempted at the instant when a particular event $e$ occurs. The preemption of $A$ by $e$ is expressed by imposing $a / e$ for all events $a$ in $A$, which asserts that no event in $A$ can occur after $e$. In other words, $e$ terminates the execution $A$. The asymmetric event structures for an Orc expression is defined by two steps.

The first step is a compositional translation of Orc expressions into mathematical structures called heaps, introduced in Section 2.2 Heaps are sets of inductively defined events, following a method originally proposed by Esparza et al [5] to encode net unfoldings. Heaps are useful for two reasons. First, they provide a concrete representation of asymmetric event structures that is suitable for effective coding of algorithms in software. Second, and more importantly, they can specify fragments of computations that refer to virtual events offered by an execution from another heap. The latter feature proved extremely useful for deriving the heap semantics of Orc, structurally.

In the second step, the heap is converted into an asymmetric event structure which is a recognized semantic domain, equipped with well defined notions of configurations to model 
partially ordered executions. A correspondence of these asymmetric event structures with the existing sequential trace semantics of Orc is also shown.

\section{Asymmetric Event Structures and Heaps}

In this section we recall the needed background on Asymmetric Event Structures (AES). Then we motivate the need for the new concept of heap and introduce it. Finally, we show how to generate AES from heaps.

\subsection{Asymmetric Event Structures with Labels}

Following 17, 1], an Asymmetric Event Structure (AES) is a model of computation consisting of a set of events and two associated binary relations, the causality relation $\preceq$ and the asymmetric conflict relation $\nearrow$. If for events $e$ and $e^{\prime}, e \preceq e^{\prime}$ holds, then $e$ must occur before $e^{\prime}$ can occur. If $e \nearrow e^{\prime}$ holds, then the occurrence of $e^{\prime}$ preempts the occurrence of $e$ in the future. Thus if both $e$ and $e^{\prime}$ occur in an execution, $e$ necessarily happens before $e^{\prime}$. In this sense, $\nearrow$ can also be seen as a "weak causality" relation.

Formally, an AES is a tuple $\mathbf{G}=(E, \preceq, \nearrow)$, where $E$ is a set of events, and $\preceq$ and $\nearrow$ are the causality and asymmetric conflict binary relations over $E$, satisfying the following conditions:

1. $\preceq$ is a partial order, and $\lfloor e\rfloor={ }_{\operatorname{def}}\left\{e^{\prime} \in E \mid e^{\prime} \preceq e\right\}$ is finite;

2. $\forall e, e^{\prime} \in E$ :

$$
\begin{array}{r}
e \prec e^{\prime} \Rightarrow e \nearrow e^{\prime} \\
\text { the restriction of } \nearrow \text { to }\lfloor e\rfloor \text { is acyclic } \\
e \#^{a} e^{\prime} \Rightarrow e \nearrow e^{\prime}
\end{array}
$$

where $\#^{a}$ is the conflict relation, which relates events that preempt each other. For two events, if $e \nearrow e^{\prime}$ and $e^{\prime} \nearrow e$ then $e \#^{a} e^{\prime}$, and only one of $e$ and $e^{\prime}$ can occur in an execution. The conflict relation finds sets of mutually conflicting events using this recursive definition:

$$
\begin{aligned}
e_{0} \nearrow e_{1} \nearrow \ldots e_{n} \nearrow e_{0} & \Rightarrow \#^{a}\left(\left\{e_{0}, \ldots, e_{n}\right\}\right) \\
{\left[\#^{a}(A \cup\{e\})\right] \wedge\left[e \preceq e^{\prime}\right] } & \Rightarrow
\end{aligned}
$$

The second condition ensures that a conflict with $e$ is inherited by all the events caused by $e$.

Given an event structure, a configuration is a set of events that obey the causality and conflict constraints, and so represent a valid execution instance of the event structure. For $\mathbf{G}=(E, \preceq, \nearrow)$ an AES, a configuration of $\mathbf{G}$ is a set $\kappa \subseteq E$ of events such that 
1. the restriction of $\nearrow$ to $\kappa$ is well-founded;

2. $\left\{e^{\prime} \in \kappa \mid e^{\prime} \nearrow e\right\}$ is finite for every $e \in \kappa$;

3. $\kappa$ is left-closed with respect to $\preceq$, i.e., $\forall e \in \kappa, e^{\prime} \in E, e^{\prime} \preceq e$ implies $e^{\prime} \in \kappa$.

For our coding of Orc, we will need to label the events. Thus we shall consider Labeled AES (LAES), which are tuples of the form $\mathbf{G}=(E, \preceq, \nearrow, \lambda)$, where $\lambda: E \mapsto \Lambda,(\Lambda$ is a set of labels) is the labeling (partial) function.

Discussion: from event structures to heaps. Asymmetric event structures allow an event to occur only if its causes have already occurred, and it is not prevented by the occurrence of some other event. This yields a simple and elegant mathematical model for complete concurrent systems that, in all its variants, comes equipped with a comprehensive categorical apparatus [1].

Although event structures work well for complete programs, they cannot easily represent fragments of behavior. Such fragments arise naturally when constructing the behavior of a program from the behaviors of the subexpressions in the program - as is the standard practice in denotational semantics. For such formalisms, structural translation of programs to (asymmetric) event structures cannot be directly achieved.

By offering the additional concept of place, Petri nets and their extensions and variants 17, 1] make structural translation easier. Explicit encoding of places allows one fragment to depend upon resources supplied by another fragment. Other features of wide area languages are not so easily supported by Petri nets; modelling dynamic creation of processes requires non-trivial extensions of nets, such as, e.g., net systems 22. These extensions require another layer of semantics to specify their executions. Therefore, using such Petri net extensions results in a complex two-stage semantics: from the formalism to, e.g., net systems, and, from net systems to their semantic domain. Such a translation was proposed in 14 for Orc, resulting in excessive formalism and complex software coding.

So, a natural idea consists in bypassing the above two-stage approach, by considering directly occurrence nets, with read arcs. To be more effective and get close to implementation, we decided in addition to use an explicit inductive coding of such occurrence nets, following the technique first proposed by Esparza et al. [5]. This results in the notion of heap described in the next section. The subclass of "effective" heaps translate immediately into asymmetric event structures.

\section{$2.2 \quad$ Heaps}

Heaps are sets of events coded in a particular form. A heap event is encoded based on the conditions that enable its occurrence. The enabling condition can either be consumed by the event or can be read and not consumed. The conditions in turn, refer to the events that created them. More precisely:

$$
\begin{aligned}
\text { event } & =(\text { consume conditions, read conditions, label }) \\
\text { condition } & =(\text { cause event }, \text { mark })
\end{aligned}
$$


where

- consume conditions is the set of conditions that are consumed by the event;

- read conditions is the set of conditions that are only read (and not consumed) by the event;

- label is a label (for our use in Orc semantics, it will be the Orc action performed by the event);

- mark is a label to distinguish different conditions created by an event.

We formalize this next.

Definition 1 Call heap a tuple $(E, C, S, A, M)$, where:

1. $E$ and $S$ are two sets of events such that $E \subseteq S, C$ is a set of conditions, $A$ is an alphabet of labels, and $M$ is a set of marks.

2. Events $e \in E$ have the following form:

$$
e=(\bullet e, \underline{e}, a)
$$

where $e \subseteq C$ and $\underline{e} \subseteq C$ are the sets of conditions consumed and read by e, respectively, and $a \in A$ is the label of $e$. We require that $\bullet e \cap \underline{e}=\emptyset$ and $\bullet e \cup \underline{e} \neq \emptyset$.

3. Conditions $c \in C$ have the following form:

$$
c=(f, \mu)
$$

where $f \in S$ and $\mu \in M$ is the mark of condition $c$.

4. $C$ and $S$ are minimal, for set inclusion, having the above properties. $S$ is called the support of $E$ and $C$ is its set of conditions.

By abuse of notation, we call $E$ alone a heap, and $C_{E}$ will denote the set of conditions associated to $E$. Throughout this paper, we distinguish a fixed event

$$
\perp=(\emptyset, \emptyset, \star)
$$

called the dummy event, where label $\star$ means the absence of label. Note that $\perp$ cannot belong to a heap, it can, however, belong to the support of a heap. Set $E_{\perp}=E \cup\{\perp\}$. For an event $e$ of the form (7), the set of conditions

$$
\stackrel{e}{e}=\bullet e \underline{e}
$$

is called the pre-set of $e$. We define the set of minimal conditions of a heap $E, \min C o n d s(E)$ to be the set

$$
\min \operatorname{Conds}(E)=_{\text {def }}\left\{(f,-) \mid(f,-) \in C_{E}, f \notin E\right\}
$$


Figure 2 shows some example heaps (for Orc expressions). The events of the heap are shown in rectangles, labelled by their corresponding Orc actions. The conditions are the circles. An event has input directed arcs from conditions consumed by it, and undirected dashed arcs from those that are read. Outgoing arcs from an event point to conditions that refer to that event. Minimal conditions refer to the $\perp$ event, which is not shown. A dashed triangle on top of a minimal condition indicates the label of an external event that the condition depends upon. Examples of external events, which are included in the support of the heap, are $e, f_{1}$, and $f_{2}$.

The conflict and read conditions within the events of a heap define constraints between events, in the style of an event structure. Given a heap $E$ we define the following relations between events in $E$ (superscript * denotes transitive closure):

$$
\begin{aligned}
& \preceq_{E}=\triangleleft^{*} \text { where } \triangleleft=\left\{(f, e) \mid f^{\bullet} \cap \underline{e}^{\prime} \neq \emptyset\right\} \cup I_{E} \\
& I_{E} \text { is the identity relation on } E \times E \\
& \nearrow_{E}^{\prime}=\prec_{E} \cup\left\{(f, e) \mid \exists e^{\prime} \in E_{\perp}, e_{1}:\left[\begin{array}{cc} 
& \left(e^{\prime},-\right) \in \cdot f \cap \bullet^{\bullet} e_{1} \\
\wedge & e_{1} \preceq_{E} e
\end{array}\right]\right\} \\
& \nearrow_{E}=\nearrow_{E}^{\prime} \cup\left\{(e, f) \mid e \#_{E}^{a} f\right\}
\end{aligned}
$$

where event variables $e, e_{1}$ and $f$ range over $E$, and the symmetric conflict relation $\#_{E}^{a}$ is deduced from $\nearrow_{E}^{\prime}$ via (45). The reason for the two-step definition of $\nearrow_{E}$ is that $\nearrow_{E}^{\prime}$ satisfies conditions (112415), but not necessarily (3). The latter is enforced by second step in the definition, from $\nearrow_{E}^{\prime}$ to $\nearrow_{E}$. Next, equip $E$ with a labeling map

$$
\alpha_{E}(e)={ }_{\text {def }} a
$$

where event $e=(\bullet e, \underline{e}, a)$. We shall denote by

$$
\min (E)=\left\{e \in E \mid \forall f \in E: f \preceq_{E} e \Rightarrow f=e\right\}
$$

the set of events $e \in E$ that are minimal for the relation $\preceq_{E}$. For readability, we omit the subscript $_{E}$ in the sequel. In the SEND heap in Figure 2$] \preceq f_{1}$ holds, where $e$ is the event labelled $M_{k 1}$ or $k 1$ ? $v_{1}$. Also $e \nearrow f_{1}$ holds for all events $e$ in the heap (except $f_{1}$ ).

Definition $2 A$ configuration of a heap $E$ is any finite subset $\kappa$ of $E$ with the following properties:

1. the restriction of $\nearrow$ to $\kappa$ is well-founded;

2. $\left\{e^{\prime} \in \kappa \mid e^{\prime} \nearrow e\right\}$ is finite for every $e \in \kappa$;

3. $\kappa$ is left-closed with respect to $\preceq$, i.e., $\forall e \in \kappa, e^{\prime} \in E, e^{\prime} \preceq e$ implies $e^{\prime} \in \kappa$;

4. for each event $e$ belonging to $\kappa$, if $f^{\bullet} \cap \underline{\bullet} \neq \emptyset$ then $f \in E_{\perp}$. 
As for AES, heap configurations represent legal executions. By condition 3 condition 4 is equivalent to requiring that $f \in \kappa$. Conditions [1] coincide with those involved in the definition of configurations for AES, see Section 2.1. Condition廿 is new. For e.g, in the SEND heap of Figure 2 any configuration having event $f_{1}$ has to include its causal predecessors, i.e the events labelled $M_{k 1}$ and $k 1$ ? $v_{1}$. Event $f_{2}$ cannot appear in such a configuration since it is in mutual conflict with $f_{1}$, thus Condition 1 would be violated.

Let $\operatorname{Configs}(E)$ be the set of all configurations of heap $E$.

\subsection{From Heaps to LAES}

One may expect $(E, \preceq, \nearrow, \alpha)$ to be an LAES. This is not true in general, as certain axioms may be violated (e.g, the causal relation $\preceq$ may not be antisymmetric, or some events may need external events for their enabling). In this section we show how to extract from any heap $E$, an effective heap which has a direct correspondence with an LAES.

Definition 3 Given a heap $E$, its effective heap $\mathcal{G}[E]$ is defined as:

$$
\mathcal{G}[E]=_{\operatorname{def}} \bigcup_{\kappa \in \operatorname{Configs}(E)} \kappa .
$$

$\mathcal{G}[E]$ possesses a subset of $E$ as its set of events. Generation of $\mathcal{G}[E]$ from a heap $E$ is by pruning and by Definition 2 This generation is constructive. The introduction of effective heap $\mathcal{G}[E]$ is justified by the following result, where symbols $\preceq, \nearrow$, and $\alpha$ are the restrictions, to $\mathcal{G}[E]$, of the relations and map defined in (9), (10), and (11), respectively.

Theorem $1 \mathcal{A}[E]=(\mathcal{G}[E], \preceq, \nearrow, \alpha)$ is an LAES. Furthermore, $\mathcal{G}[E]$ is the maximal subset of events of $E$ that induces an LAES.

Proof Outline. The complete proof is given in Appendix A The first part is proved by using (9), (10) and Definition 2 to show that relations $\preceq, \nearrow$ on $\mathcal{G}[E]$ satisfy the conditions required for a LAES. The second part is proved by showing that any configuration of a maximal LAES induced by $\mathrm{E}$ is contained in $\operatorname{Configs}(E)$ and thus in $\mathcal{G}[E]$.

Remark: The reader should not confuse between the notion of heaps given here and those in 11, 10, where the authors study heaps formed by blocks representing durations of executions in transition systems. Since their heaps are downward causally closed conflict free partial orders, they correspond to configurations in our setting, rather than the heaps in the above sense.

\subsection{Generic Operations on Heaps}

We list here a few operations on heaps that are useful for wide area computing. From now on, we specialize marks to being lists, with the usual operations.

- Marking: Marking creates distinct copies of a heap. For a heap $E$ and $m$ a mark, $E^{m}$ is the heap where symbol $m$ has been appended to the mark $\mu(c)$ of each condition $c \in \min \operatorname{Conds}(E)$. The recursive definitions of events and conditions in $E$ ensures that this operation creates a new instance of $E$. 
- Disjoint Union: The disjoint union of heaps $E$ and $F$ where left and right are fixed marks is:

$$
E \uplus F={ }_{\text {def }} \quad E^{l e f t} \cup F^{\text {right }}
$$

- Preemption: For a heap $E$ and $F \subseteq E$, the preemption of $E$ by $F$ terminates execution of $E$ when any event in $F$ occurs. Formally, $\operatorname{STOP}_{F}(E)$ is the heap obtained by replacing each event $e=(\bullet e, \underline{e}, a)$ of $E$ by the following event $\varphi(e)$ :

$$
\varphi(e)==_{\operatorname{def}} \begin{cases}(\bullet e \cup\{(\perp, \text { stop })\}, \underline{e}, a) & \text { if } e \in F . \\ (\bullet e, \underline{e} \cup\{(\perp, \text { stop })\}, a) & \text { if } e \notin F .\end{cases}
$$

- Copy: For two heaps $E$ and $F$, we define $\operatorname{COPY}_{l}(E, F)$ to be a copy of $E$ with respect to context heap $F$. For a mark $l, \mathrm{COPY}_{l}(E, F)$ is a fresh heap obtained by changing all minimal conditions $(e, \mu) \in \min \operatorname{Conds}(E)$ as follows:

$$
(e, \mu)= \begin{cases}(e,(\mu, l)) & \text { if }(e, \mu) \notin C_{F} \\ (e, \mu) & \text { if }(e, \mu) \in C_{F}\end{cases}
$$

where $C_{F}$ is the set of associated conditions of the context heap $F$. Intuitively, events in $E$ may share conditions (and thus are related) with events in the context heap $F$. The copy of $E$ with respect to context $F$ keeps these conditions intact in the copy to preserve the relations between the copied events and those in $F$.

\section{Orc Syntax and Semantics}

The reader is referred to 12 for an introduction to and motivation for Orc, as a language for wide area computing. The syntax and operational semantics of Orc in the form of SOS rules [7, are given in Figure [1

An Orc expression $f$ can perform action $a$ and transform itself into the expression $f^{\prime}$, which is denoted by the transition $f \stackrel{a}{\rightarrow} f^{\prime}$. The actions $A$ and values $V$ are described by the following grammar:

$$
\begin{array}{ll}
a \in A & ::=M_{k}(v)|k ? v| ! v|\tau| \tau_{v} \\
v \in V & ::=x\left|v_{k}\right| \mathbf{v}
\end{array}
$$

The actions $A$ are the transition labels of the Orc operational semantics, except for the $\tau_{v}$ action which is an intermediary action needed for creating heaps. The $x$ are variable names. They are placeholders for the value which will eventually replace that variable in the expression. The return values $v_{k}$ are indexed by call handles. They are placeholders for the values returned from site calls. The ground values $\mathbf{v}$ are the constant values which are always available. 


$$
\begin{aligned}
& f, g, h \in \text { Expression }::=M(p)|E(p)| f|g| f>x>g \mid f \text { where } x: \in g \mid ? k \\
& p \in \text { Actual } \quad::=x \mid v \\
& \text { Definition }::=E(x) \underline{\Delta} f \\
& \frac{k \text { fresh }}{M(v) \stackrel{M_{k}(v)}{\longrightarrow} ? k} \\
& \text { (SiteCall) } \\
& \frac{f \stackrel{a}{\rightarrow} f^{\prime} \quad a \neq ! v}{f>x>g \stackrel{a}{\rightarrow} f^{\prime}>x>g} \\
& ? k \stackrel{k ? v}{\longrightarrow} \operatorname{let}(v) \\
& \text { (SiteRet) } \\
& \frac{f \stackrel{! v}{\rightarrow} f^{\prime}}{f>x>g \stackrel{\tau}{\rightarrow}\left(f^{\prime}>x>g\right) \mid[v / x] . g} \\
& \operatorname{let}(v) \stackrel{! v}{\longrightarrow} 0 \\
& \frac{f \stackrel{a}{\rightarrow} f^{\prime}}{f\left|g \stackrel{a}{\rightarrow} f^{\prime}\right| g} \\
& \frac{f \stackrel{a}{\rightarrow} f^{\prime}}{f \text { where } x: \in g \stackrel{a}{\rightarrow} f^{\prime} \text { where } x: \in g} \\
& \frac{g \stackrel{! v}{\longrightarrow} g^{\prime}}{f \text { where } x: \in g \stackrel{\tau}{\rightarrow}[v / x] . f} \\
& \frac{g \stackrel{a}{\rightarrow} g^{\prime}}{f|g \stackrel{a}{\rightarrow} f| g^{\prime}} \\
& \frac{g \stackrel{a}{\rightarrow} g^{\prime} \quad a \neq ! v}{f \text { where } x: \in g \stackrel{a}{\rightarrow} f \text { where } x: \in g^{\prime}} \\
& \frac{\llbracket E(x) \triangleq f \rrbracket \in D}{E(p) \stackrel{\tau}{\rightarrow}[p / x] . f}
\end{aligned}
$$

Figure 1: The Syntax (top) Operational Semantics (bottom) of Orc

Observe the following. Due to rule (Def), recursive definitions are possible in Orc. Also, rule (Asym1V) exhibits termination of $g$ upon its first publication.

To simplify the translation, we assume that the Orc programs we consider have distinct variable names. This restriction does not reduce the program's expressivity and can be enforced by a simple syntactic pre-processing step.

\section{Denotations for Orc Expressions}

In this section, we show how to construct the heap of an Orc program, and then its LAES. We begin with further useful operations on heaps that are specific to Orc. Then, we provide the heap semantics of Orc base expressions and operators. 
- Free Variables: $E(x)$ is the set of all events in heap $E$ which depend on $x$.

$$
E(x)=\left\{e \in E \mid \exists e^{\prime} \in E, e^{\prime} \preceq_{E} e, \alpha\left(e^{\prime}\right) \in\left\{M_{k}(x), ! x, \tau_{x}\right\}\right\}
$$

Call $x$ a free variable of $E$ if $E(x)$ is nonempty. Let $E(\bar{x})$ be the events in $E$ that do not depend on $x: E(\bar{x})=E-E(x)$.

- Publication events: !E is the set of publication events of heap $E$ :

$$
! E=\{e \mid \alpha(e)=! v\}
$$

- Preemption: Stopping $E$ after the first value publication is defined as:

$$
\operatorname{STOP}(E) \quad=_{\text {def }} \quad \operatorname{STOP}_{E}(E)
$$

- Send: For a publication event $e=(\bullet e, \underline{e}, ! v)$, define the $\tau(e)$ to be the event obtained by changing the label of $e$ as follows:

$$
\alpha(e)= \begin{cases}\tau_{x} & \text { if } \alpha(e)=! x, \text { for any variable } x \\ \tau & \text { otherwise }\end{cases}
$$

The heap $\operatorname{SEnD}(E)$ is the heap $E$ where all the publication events $e$ in $E$ are replaced by $\tau(e)$. The publication events are still identifiable by their marks.

- Link: For a heap $E$, a context heap $C$, an event $f$ not belonging to $E$, and a value $v$,

$$
\operatorname{LINK}(f, v, x, E, C)
$$

is a heap in which variable $x$ is bound to value $v$ after external event $f$. The context heap $C$ identifies parts of $E$ that are not affected by the variable binding. $\operatorname{LINK}(f, v, x, E, C)$ is the heap resulting from the following operations:

1. Create $E^{\prime}=\operatorname{COPy}_{f}(E, C)$ a new copy of $E$ with respect to context heap $C$ and marked with label $f$. In making this copy, each event $e \in E$ has a unique corresponding event $e^{\prime}=\varphi_{f}(e) \in E^{\prime}$.

2. Change all $e^{\prime}=\left(\bullet e^{\prime}, \underline{e}^{\prime}, a\right) \in E^{\prime}$ as below, where $e=\varphi_{f}^{-1}\left(e^{\prime}\right)$ :

$$
e^{\prime}= \begin{cases}\left(\bullet e^{\prime} \cup\{(f, e)\}, \underline{e}^{\prime},[v / x] a\right) & \text { if } e^{\prime} \in \min \left(E^{\prime}\right) \\ \left(\bullet e^{\prime}, \underline{e}^{\prime},[v / x] a\right) & \text { if } e^{\prime} \notin \min \left(E^{\prime}\right)\end{cases}
$$

The substitution $[v / x] a$ replaces the variable $x$ by $v$ in the action $a$. If the variable $x$ does not occur in $a$, the substitution leaves $a$ unchanged. In the heap constructed here, the event $f$ referred by $e^{\prime} \in \min \left(E^{\prime}\right)$ is not in the heap. 
- Receive: We next construct a heap that can receive any values that is published by another heap. If $e$ is a publication event, $\tau(e)$ is the event $e$ with its action changed according to (15). We define

$$
\operatorname{RECV}_{x}(E, F, C)=\bigcup_{f \in ! E, \alpha(f)=! v} \operatorname{LiNK}(\tau(f), v, x, F, C)
$$

Observe that, if !E is empty, this yields $\operatorname{RECV}_{x}(E, F, C)=\emptyset$.

- Pipe: The pipe operator allows $G$ to receive publications from $F$, subject to a context $C$ that identifies parts of $G$ not affected by the communication.

$$
\operatorname{PIPE}_{x}(F, G, C)=\operatorname{SEND}(F) \cup \operatorname{RECV}_{x}(F, G, C)
$$

\subsection{Heaps of Base Expressions}

For an Orc expression $f,[f]$ is its heap denotation. In the following, nil is a distinguished symbol indicating the absence of mark.

$$
\begin{aligned}
{[\mathbf{0}]=} & \emptyset \\
{[\operatorname{let}(v)]=} & \{(\{c\}, \emptyset, ! v)\} \\
& \text { where condition } c=(\perp, n i l) \\
{[? k]=} & \left\{e=\left(\left\{c_{1}\right\}, \emptyset, k ? v_{k}\right),\left(\left\{c_{2}\right\}, \emptyset, ! v_{k}\right)\right\} \\
& \text { where condition } c_{1}=(\perp, n i l), c_{2}=(e, n i l) \\
{[M(v)]=} & \left\{e=\left(\left\{c_{1}\right\}, \emptyset, M_{k}(v)\right), f=\left(\left\{c_{2}\right\}, \emptyset, k ? v_{k}\right),\left(\left\{c_{3}\right\}, \emptyset, ! v_{k}\right)\right\} \\
& \text { where condition } c_{1}=(\perp, n i l), c_{2}=(e, n i l), c_{3}=(f, n i l), \\
& k \text { is fresh. } \\
& {[[v / x] f] } \\
& \text { where } E \text { is an expression definition and } E(x) \triangleq f
\end{aligned}
$$

\subsection{Heaps for the Combinators}

$$
\begin{aligned}
{[f \mid g]=} & {[f] \uplus[g] } \\
{[f>x>g]=} & \operatorname{PIPE}_{x}([f],[g], \emptyset) \\
{[g \text { where } x: \in f]=} & \operatorname{PIPE}_{x}(\operatorname{STOP}(F), G(x), G(\bar{x})) \cup G(\bar{x}) \\
& \text { where } F=[f]^{\text {right }} \text { and } G=[g]^{\text {left }}
\end{aligned}
$$

Figure 2 gives the intermediary and the final heap for the Orc expression $\{\operatorname{let}(1) \gg S(x)\}$ where $x: \in\{M \mid N\}$. Note the two publications $f_{1}$ and $f_{2}$, by the parallel 

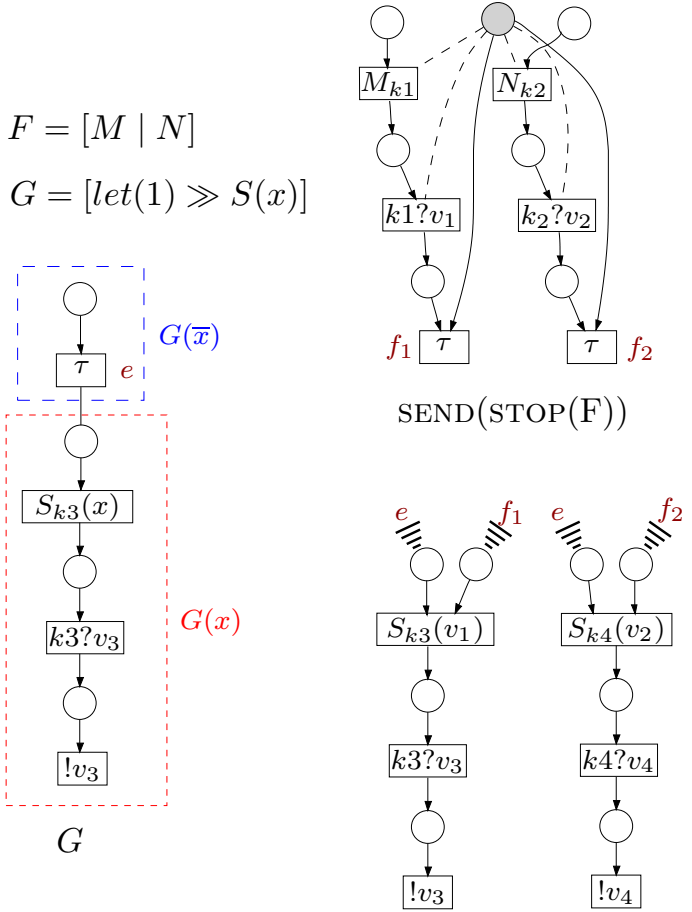

$\operatorname{RECV}_{x}(\operatorname{STOP}(F), G(x), G(\bar{x}))$

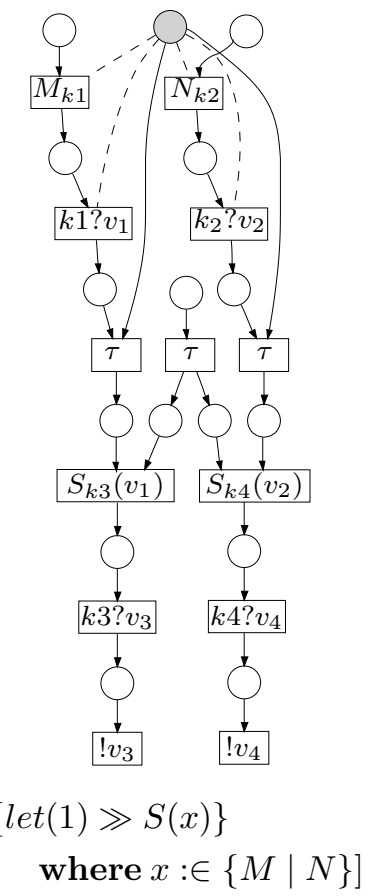

where $x: \in\{M \mid N\}]$

Figure 2: Heap Construction Example: The shaded condition is the $(\perp, s t o p)$ condition introduced by the STOP operator. A dashed arrowhead to a minimal condition of the RECV heap from an event name states that the condition depends on that external event. The external events here are $e$ and $f_{1}, f_{2}$ in heaps $G(\bar{x})$ and $\operatorname{SEND}(\operatorname{STOP}(F))$ respectively. When these heaps are combined in the rightmost heap, these events become internal events.

composition $M \mid N$. These are made conflicting by the extra (shaded) condition created by the STOP operator.

Following Section 2.3 we can now translate the heaps associated to Orc expressions into LAES. The LAES of an expression $f$ is $\llbracket f \rrbracket=\mathcal{A}[[f]]$.

\subsection{Recursive Definitions}

The treatment of recursive definitions follows that given in [7, except that the denotation of an expression $f$ is the heap $[f]$ instead of the set of traces $\langle f\rangle$. The heap for a recursive Orc definition $f \underline{\Delta} \operatorname{Exp}(f)$ is the limit of a series of increasing approximations $0 \sqsubseteq \operatorname{Exp}(0) \sqsubseteq$ 
$\operatorname{Exp}(\operatorname{Exp}(0)) \sqsubseteq \ldots$ To ensure existence of the limit, the least fixpoint of $\operatorname{Exp}$, we show that the Orc combinators are monotonic with respect to $\sqsubseteq$. For $F$ and $G$ two heaps, define

$$
F \prec G \quad \text { if } \quad F \subseteq G \text { and } C_{F} \cap C_{G-F}=\emptyset
$$

Then for Orc expressions, $f \sqsubseteq g$ if $[f] \prec[g]$. The motivation for having the second condition in (20) is that it is needed in the proof of Lemma 2 below.

Lemma 1 Relation $\prec$ is a partial order on heaps.

Proof. Assume $F \prec G \prec H$. So $C_{F} \cap C_{G-F}=\emptyset$ and $C_{G} \cap C_{H-G}=\emptyset$. Writing $H-F=$ $(H-G) \cup(G-F)$, we have $C_{F} \cap C_{H-F}=\left(C_{F} \cap C_{H-G}\right) \cup\left(C_{F} \cap C_{G-F}\right)$. The second term is an empty set. Since $F \subseteq G$, we have $C_{F} \subset C_{G}$. This gives $C_{F} \cap C_{H-G}=\emptyset$ which ensures $F \prec H$ and proves the lemma.

Lemma 2 The Orc combinators are monotonic in both arguments. In particular, given $f \sqsubseteq g$, then

$$
\begin{aligned}
f \mid h & \sqsubseteq g \mid h \\
f>x>h & \sqsubseteq g>x>h \\
h>x>f & \sqsubseteq h>x>g \\
f \text { where } x: \in h \quad & \sqsubseteq g \text { where } x: \in h \\
h \text { where } x: \in f & \sqsubseteq h \text { where } x: \in g
\end{aligned}
$$

Proof sketch: (Complete proof in Appendix C). These conditions are established by examining the corresponding constructions on heaps. Monotonicity of most operators can be established by inspection, since they are defined as pointwise functions on the individual events in a heap. One special case is the $\mathrm{COPY} \operatorname{COPY}_{l}(E, F)$ is not monotonic in its second argument: although $\emptyset \prec F$, it is easy to see that $\operatorname{COPY}_{l}(E, \emptyset) \nprec \operatorname{COPY}_{l}(E, F)$ in general. However, from Section 4.2 we see that we only need monotonicity of the special case where the arguments to COPY are the partition $G(x), G(\bar{x})$ of $G$. Assume $G \prec G^{\prime}$ and set $H=G^{\prime}-G$. We have

$$
\begin{aligned}
\mathrm{COPY}_{l}\left(G^{\prime}(x), G^{\prime}(\bar{x})\right) & =\mathrm{COPY}_{l}\left(G(x) \cup H(x), G^{\prime}(\bar{x})\right) \\
& =\mathrm{COPY}_{l}\left(G(x), G^{\prime}(\bar{x})\right) \cup \mathrm{COPY}_{l}\left(H(x), G^{\prime}(\bar{x})\right) \\
& =\mathrm{COPY}_{l}(G(x), G(\bar{x}) \cup H(\bar{x})) \cup \mathrm{COPY}_{l}\left(H(x), G^{\prime}(\bar{x})\right)
\end{aligned}
$$

By definition of the copy, $\mathrm{COPY}_{l}(G(x), G(\bar{x}) \cup H(\bar{x}))$ is obtained by changing all minimal conditions $c=(e, \mu) \in \operatorname{minConds}(G(x))$ as specified in (14). By the second condition of (20), we have minConds $(G) \cap C_{H}=\emptyset$. Thus $\operatorname{COPY}_{l}(G(x), G(\bar{x}) \cup H(\bar{x}))=\operatorname{COPY}_{l}(G(x), G(\bar{x}))$, and thus implies that $\quad \operatorname{COPY}_{l}(G(x), G(\bar{x})) \quad$ $\operatorname{COPY}_{l}\left(G^{\prime}(x), G^{\prime}(\bar{x})\right)$. 


\section{Correctness of Orc heap semantics}

In this section we prove the correctness of the heap semantics for Orc. We do this by showing that the heap semantics is equivalent to an interleaving trace semantics for Orc, developed in 7. The trace $\langle f\rangle$ of an Orc expression $f$ is a set of the sequence of actions that it can perform. Such a sequence is derived from the labels of successive transitions (according to Figure 10 that $f$ can perform. An additional event, called substitution event is introduced to define traces of expressions with free variables. The corresponding rule is

$$
f \stackrel{[v / x]}{\longrightarrow}[v / x] . f
$$

which replaces occurrence of variable $x$ in $f$ by the value $v$.

For the inductive proof, we need to define how configurations of a heap $[f]$ representing an Orc expression $f$ are mapped to traces of $\langle f\rangle$. Since our heap semantics does not introduce substitution events, we need to capture them indirectly. This is done in several steps.

1. We first prepare every heap $E$ as follows: let $X$ be a finite set of free variables containing the set $X_{E}$ of all free variables of $E$. For every $x \in X$, let $e_{x}$ be an additional event not belonging to $E$, defined as follows:

$$
e_{x}=\left(\{c\}, \emptyset, \sigma_{x}\right), \text { where } c=(\perp, x)
$$

Then, for every event $e=(\bullet e, \underline{e}, a) \in E$, define the event $e^{\prime}=\left(\bullet e^{\prime}, \underline{e}^{\prime}, a\right)$ where:

$$
\text { - } e^{\prime}=\bullet e \cup\left\{\left(e_{x}, e\right) \mid x \in X \text { and } e \in E(x)\right\} \text {, and } \underline{e}^{\prime}=\underline{e}
$$

Let $E_{X}=\Phi_{1}^{X}(E)=\left\{e^{\prime} \mid e \in E\right\} \cup\left\{e_{x} \mid x \in X\right\}$ be the resulting heap. Each event $e_{x}$ is concurrent to $E_{X}-E_{X}(x)$ and precedes $E_{X}(x)$. All $e_{x}$ events are concurrent with each other. Each configuration $\kappa$ of $E$ gives rise to a set $K_{X}(\kappa)$ of configurations of $E_{X}$ through the previously defined map $e \mapsto e^{\prime}$. Every $\kappa_{X} \in K_{X}(\kappa)$ is obtained by adding, to the image $\kappa^{\prime}$ of $\kappa$, every $e_{x}$ such that $\kappa(x) \neq \emptyset$, plus possibly additional ones, depending on $X$.

2. For this step, set $X$ is fixed. Map each configuration $\kappa_{X}$ of $E_{X}$ to the set of all its linear extensions:

$$
\kappa_{X} \mapsto \ell\left(\kappa_{X}\right)=\left\{\widehat{t} \mid \widehat{t} \text { is a linear extension of } \kappa_{X}\right\}
$$

Then, to every $\widehat{t} \in \ell\left(\kappa_{X}\right)$, we associate the following set of traces:

$$
\widehat{t} \mapsto \mathcal{E}(\widehat{t})
$$

where $\mathcal{E}(\widehat{t})$ is the set of all traces obtained as follows: for every value $v$ and every $e_{x}$ belonging to $\widehat{t}$ :

(a) replace, in $e_{x}$, action label $\sigma_{x}$ by substitution $\left[v_{x} / x\right]$; 
(b) substitute $v_{x}$ for $x$ in all actions of $\widehat{t}$ where $x$ occurs;

(c) replace each event by its associated action.

By abuse of notation, for $\widehat{T}$ a set of traces, we also write $\mathcal{E}(\widehat{T})=\bigcup_{\widehat{t} \in \widehat{T}} \mathcal{E}(\widehat{t})$, so that $\mathcal{E} \circ \ell\left(\kappa_{X}\right)$ is well defined. Denote the operations of this step by the map

$$
\Phi_{2}(\kappa)=\mathcal{E} \circ \ell(\kappa)
$$

3. We finally set

$$
\Phi_{3}^{X}(E)=\bigcup_{\kappa \in \operatorname{Configs}\left(\Phi_{1}^{X}(E)\right)} \Phi_{2}(\kappa) \text { and } \Phi(E)=\bigcup_{X \supseteq X_{E}} \Phi_{3}^{X}(E)
$$

Theorem 2 (Semantic equivalence) For every Orc expression $f$, we have

$$
\langle f\rangle=\Phi([f])
$$

Proof. The proof is by structural induction over Orc expression $f$. See appendix D.3

\section{Related Work}

Closest to our present study is the work and 14, where Orc expressions are translated to colored Petri net systems [2]. Another closely related work is reported in Bruni et al. [3], where the authors link the Orc language to Petri nets and the Join Calculus; it is advocated that Join Calculus, by offering means to support dynamic creation of names and activities as well as pruning associated with asymmetric conflict, is an adequate formalism for orchestrations. For an approach that focuses on temporal properties without partial orders nor performance evaluation, see [4, where a Timed Automaton semantics of Orc is given and used for verification purposes using the Uppaal tool. On the other hand, a number of papers have been devoted to the Petri net semantics of the most widely used language for orchestration, namely BPEL, see [6, 9, 13, 15, 8] and the tutorial [16].

Our work is unique in that it provides a direct coding of a wide area computing language into asymmetric event structures. This is of immediate use in QoS studies, as the latter builds on timed and/or probabilistic enhancements of partial order models [14.

\section{Conclusion}

We have presented a partial order semantics for Orc, a structured orchestration language with support for termination and recursive process instantiation. The semantics uses heaps to encode sets of interrelated events because they simplify manipulation of the fragments of program behavior that arise when analyzing the sub-expressions of a program. These 
fragments are composed to create effective heaps, from which more traditional asymmetric event structures are derived. We show that the event structure semantics is equivalent to a previous denotational trace semantics.

The heap semantics provides a model of true concurrency and also directly support analysis of non-functional properties of Orc programs, including critical path and dependency analysis that can affect Quality of Service.

A verbatim coding of the Orc heap semantics has been written in Prolog - it takes only two pages of Prolog code. Based on this tool, an analysis of Quality of Service is being developed. Results related to this more applied work will be presented elsewhere.

\section{References}

[1] Paolo Baldan, Andrea Corradini, and Ugo Montanari. Contextual Petri nets, Asymmetric Event Structures, and Processes. Inf. Comput., 171(1):1-49, 2001.

[2] Eike Best, Raymond R. Devillers, and Maciej Koutny. The Box Algebra = Petri Nets + Process Expressions. Inf. Comput., 178(1):44-100, 2002.

[3] Roberto Bruni, Hernán C. Melgratti, and Emilio Tuosto. Translating Orc Features into Petri Nets and the Join Calculus. In WS-FM, pages 123-137, 2006.

[4] Jin Song Dong, Yang Liu, Jun Sun, and Xian Zhang. Verification of Computation Orchestration via Timed Automata. In ICFEM, pages 226-245, 2006.

[5] Javier Esparza, Stefan Römer, and Walter Vogler. An improvement of McMillan's Unfolding Algorithm. Formal Methods in System Design, 20(3):285-310, 2002.

[6] Sebastian Hinz, Karsten Schmidt, and Christian Stahl. Transforming BPEL to petri nets. In Business Process Management, pages 220-235, 2005.

[7] David Kitchin, William R. Cook, and Jayadev Misra. A Language for Task Orchestration and its Semantic Properties. In Proc. of the Intl. Conf. on Concurrency Theory (CONCUR), 2006.

[8] Mariya Koshkina and Franck van Breugel. Modelling and verifying web service orchestration by means of the concurrency workbench. SIGSOFT Softw. Eng. Notes, 29(5):1-10, 2004.

[9] Niels Lohmann, Peter Massuthe, Christian Stahl, and Daniela Weinberg. Analyzing Interacting BPEL Processes. In Business Process Management, pages 17-32, 2006.

[10] Jean Mairesse and T. Bousch. Asymptotic height optimization for topical IFS, Tetris heaps, and the finiteness conjecture. J. Amer. Math. Soc, 15:77-111, 2002.

[11] Jean Mairesse and Stéphane Gaubert. Modeling and Analysis of Timed Petri nets using Heaps of Pieces. IEEE Trans. Autom. Control, 44(4):683-697, 1999. 
[12] Jayadev Misra and William R. Cook. Computation orchestration: A basis for widearea computing. Journal of Software and Systems Modeling, May, 2006. Available for download at http://dx.doi.org/10.1007/s10270-006-0012-1.

[13] Chun Ouyang, Eric Verbeek, Wil M. P. van der Aalst, Stephan Breutel, Marlon Dumas, and Arthur H. M. ter Hofstede. WofBPEL: A Tool for Automated Analysis of BPEL Processes. In ICSOC, pages 484-489, 2005.

[14] Sidney Rosario, Albert Benveniste, Stefan Haar, and Claude Jard. Foundations for Web Services Orchestrations: functional and QoS aspects. In Proceedings International Symposium on Leveraging Applications of Formal Methods, Verification and Validation (ISoLA), 2006.

[15] Franck van Breugel and Mariya Koshkina. Dead-Path-Elimination in BPEL4WS. In Proceedings of the 5th International Conference on Application of Concurrency to System Design (ACSD), pages 192-201, 2005.

[16] Franck van Breugel and Mariya Koshkina. Models and Verification of BPEL. Available at http://www.cse.yorku.ca/ franck/research/drafts/tutorial.pdf, 2006. York University.

[17] Glynn Winskel. Event structures. In Advances in Petri Nets, pages 325-392, 1986. 


\section{A Proof of Theorem [1]}

We show that $(\mathcal{G}[E], \preceq, \nearrow, \alpha)$ satisfies all the conditions for an LAES given in section 2.1 Let us first show that $\preceq$ is a partial order on $\mathcal{G}[E]$. By (9]) and Condition 4 of Definition 2 $\preceq$ is a preorder on $\mathcal{G}[E]$. It thus remains to show that there exists no non trivial circuit $e_{1} \preceq e_{2} \preceq \ldots \preceq e_{n} \preceq e_{1}$. Let $\kappa$ be a configuration containing $e_{1}$. By Condition 3 of Definition 2 the circuit $e_{1} \preceq e_{2} \preceq \ldots \preceq e_{n} \preceq e_{1}$ must be contained in $\kappa$. But, since $\prec \subseteq \nearrow$, we have $e_{1} \nearrow e_{2} \nearrow \ldots \nearrow e_{n} \nearrow e_{1}$, which contradicts Condition 1 of Definition 2 This shows that $\preceq$ is a partial order on $\mathcal{G}[E]$. Also $\lfloor e\rfloor=_{\operatorname{def}}\left\{e^{\prime} \mid e^{\prime} \preceq e\right\}$ is finite, since an infinite $\prec$ sequence of events would be an infinite $\nearrow$ sequence of events, again contradicting Condition 1 of Definition 2 The same reasoning shows that $\nearrow_{\lfloor e\rfloor}={ }_{\operatorname{def}}\left\{\left(e_{1}, e_{2}\right) \mid e_{1}, e_{2} \in\lfloor e\rfloor, e_{1} \nearrow e_{2}\right\}$ is acyclic. This proves the first statement of the theorem.

To prove the second statement, let $F \subseteq E$ be such that $(F, \preceq, \nearrow, \alpha)$ is an LAES. Denote by $\kappa_{F}$ a generic configuration of this LAES. By the definition of configurations, for LAES, any such $\kappa_{F}$ must satisfy Conditions 13 of Definition 2] In addition, by (9)- (10), $\kappa_{F}$ must be such that, for each event $e$ belonging to $\kappa_{F}$, if $f^{\bullet} \cap \cap^{\bullet} \neq \emptyset$ then $f \in F$. Since $F \subseteq E$, this implies that $\kappa_{F}$ also satisfies Condition 4 of Definition 2 Hence $\kappa_{F}$ satisfies all conditions of Definition 2 for heap configurations. This proves the theorem.

\section{B Characteristic property of the Stop operator}

The following result shows that STOP is a preemption operator.

Lemma 1 Let $E$ be a heap such that $(\perp$, "stop" $) \notin C_{E}$ and let $F \subseteq E$. Let bijection $\varphi^{-1}$ be the inverse map of $\varphi$ introduced in (13) for the definition of the $\operatorname{STOP}_{F}(E)$, i.e for all $e \in E$,

$$
\varphi^{-1}(e)= \begin{cases}(\bullet e-\{(\perp, " s t o p ")\}, \underline{e}, \alpha(e)) & \text { if } e \in F \\ (\bullet e, \underline{e}-\{(\perp, " s t o p ")\}, \alpha(e)) & \text { if } e \notin F\end{cases}
$$

If $\kappa$ is a configuration of $\operatorname{STOP}_{F}(E)$, then the following properties hold:

1. $\varphi^{-1}(\kappa)$ is a configuration of $E$.

2. $\varphi^{-1}(\kappa) \cap F$ contains at most one event; if $\varphi^{-1}(e)$ is such an event, then $\forall f \in \kappa \Rightarrow$ $\neg[e \prec f]$.

Proof. The first statement is immediate, since $\varphi^{-1}$ removes read and consume conditions from the preset of each event.

To prove the second statement, assume that $\varphi^{-1}(\kappa) \cap F$ contains two events $e$ and $e^{\prime}$. Since $e, e^{\prime} \in F$, the events $\varphi(e)$ and $\varphi\left(e^{\prime}\right)$ have condition ( $\perp$, "stop") in their consume preconditions set. From (10), we have that $\varphi(e) \nearrow \varphi\left(e^{\prime}\right)$ and $\varphi\left(e^{\prime}\right) \nearrow \varphi(e)$, which imply that they cannot both occur in the same configuration $\kappa$. Now let $e \in \varphi^{-1}(\kappa) \cap F$ for a configuration $\kappa$. Following our previous argument, $\varphi(e)$ has the condition ( $\perp$, "stop") in its 
consume preconditions set. By definition of STOP, all events $f$ in $\operatorname{STOP}_{F}(E)$, and hence in $\kappa$ have $(\perp$, "stop") in their preconditions set $\bullet$. From (10) it follows that $f \nearrow e$ which implies $\neg[e \prec f]$ since $e, f$ are in the same configuration $\kappa$.

\section{Proof of Lemma 2}

These conditions are established by examining the corresponding constructions on heaps. The parallel expression $f \mid h$ is monotonic because $\uplus$ is monotonic. $\uplus$ is monotonic because marking $E^{l}$ is monotonic and $\cup$ is monotonic. Marking is monotonic because it is a pointwise function over minConds, a monotonic selection of a subset of its argument events.

Sequential composition $f>x>h$ is monotonic because $\operatorname{PIPE}_{x}(E, F, \emptyset)$ is monotonic in both $E$ and $F$. $\operatorname{PIPE}_{x}(E, F, \emptyset)$ is monotonic if $\operatorname{SEnd}(F)$ and $\operatorname{RECV}_{x}(E, F, \emptyset)$ are monotonic. SEND, like marking, is a pointwise function over a monotonic selection $! E$ of events from $E$. Receive $\operatorname{RECV}_{x}(E, F, \emptyset)$ is trivially monotonic in $E$, because it is a union over the monotonic subset $! E$, and is monotonic in $F$ if $\operatorname{LinK}(e, v, x, F, \emptyset)$ is monotonic in $F$. Linking depends on monotonicity of $\operatorname{COPY}_{l}(F, \emptyset)$, a simple pointwise function on events. Linking also applies a pointwise function based on min, a monotonic subset of a heap.

Monotonicity of asymmetric composition $f$ where $x: \in g$ is more complicated. It depends on monotonicity of $G(\bar{x})$ and $\operatorname{PIPE}_{x}(\operatorname{STOP}(F), G(x), G(\bar{x}))$. The free variable constructs, $G(x)$ and $G(\bar{x})$ are pointwise selectors of events, so they are monotonic. STOP $(E)$ is also a pointwise function affecting ! $E$, a monotonically increasing subset of E. Finally, there is the question of the monotonicity of $\operatorname{PIPE}_{x}(F, G(x), G(\bar{x}))$. As mentioned above, $\operatorname{PIPE}_{x}$ is monotonic in its first argument, in this case $F$. Monotonicity of PIPE $x$ for $G$ depends on monotonicity of $\operatorname{Link}(e, v, x, G(x), G(\bar{x}))$, which in turn depends on monotonicity of $\operatorname{COPY}_{l}(G(x), G(\bar{x}))$. Note that $\mathrm{COPY}_{l}(E, F)$ is not monotonic in its second argument: although $\emptyset \prec F$, it is easy to see that $\mathrm{COPY}_{l}(E, \emptyset) \nprec \operatorname{COPY}_{l}(E, F)$ in general. However, we only need monotonicity of the special case where the arguments to COPY are the partition $G(x), G(\bar{x})$ of $G$. Assume $G \prec G^{\prime}$ and set $H=G^{\prime}-G$. We have

$$
\begin{aligned}
\mathrm{COPY}_{l}\left(G^{\prime}(x), G^{\prime}(\bar{x})\right) & =\mathrm{COPY}_{l}\left(G(x) \cup H(x), G^{\prime}(\bar{x})\right) \\
& =\mathrm{COPY}_{l}\left(G(x), G^{\prime}(\bar{x})\right) \cup \mathrm{COPY}_{l}\left(H(x), G^{\prime}(\bar{x})\right) \\
& =\mathrm{COPY}_{l}(G(x), G(\bar{x}) \cup H(\bar{x})) \cup \mathrm{COPY}_{l}\left(H(x), G^{\prime}(\bar{x})\right)
\end{aligned}
$$

By definition of the copy, $\mathrm{COPY}_{l}(G(x), G(\bar{x}) \cup H(\bar{x}))$ is obtained by changing all minimal conditions $c=(e, \mu) \in \operatorname{minConds}(G(x))$ as specified in (14). By the second condition of (20), we have $\min \operatorname{Conds}(G) \cap C_{H}=\emptyset$. Thus $\mathrm{COPY}_{l}(G(x), G(\bar{x}) \cup H(\bar{x}))=\mathrm{COPY}_{l}(G(x), G(\bar{x}))$, and thus implies that $\operatorname{COPY}_{l}(G(x), G(\bar{x}))$ $\operatorname{COPY}_{l}\left(G^{\prime}(x), G^{\prime}(\bar{x})\right)$. This finishes the proof of Lemma 2 


\section{Proof of Correspondence Theorem 2}

\section{D.1 Definition of Terms}

\section{D.1.1 Linear extension of a trace}

Consider an execution which is a sequence of events $e_{1}, \ldots e_{n}$. The trace $t$ of this execution $\mathcal{E}\left(e_{1}, \ldots e_{n}\right)$ is the sequence of corresponding actions. Let event $e$ be such that $\forall c \in{ }^{\bullet}$, $\exists 1 \leq i \leq n$ such that $c=\left(e_{i},-\right)$. Then all the events causally preceding $e$ appear in the trace $t$. Let $1 \leq k \leq n$ be the highest index of these predecessor events. Define the linear extension of such a trace $t$ w.r.t $e, t / e$ as the set of traces

$$
t / e=\left\{\begin{array}{c}
\mathcal{E}\left(e_{1}, \ldots e_{k}, e, e_{k+1}, \ldots e_{n}\right) \\
\mathcal{E}\left(e_{1}, \ldots e_{k}, e_{k+1}, e, \ldots e_{n}\right) \\
\vdots \\
\mathcal{E}\left(e_{1}, \ldots e_{k}, e_{k+1}, \ldots e_{n}, e\right)
\end{array}\right.
$$

If we lift the definition of linear extensions of a trace to linear extensions of a set of traces, we have for a configuration $\kappa \in \operatorname{Configs}\left(\Phi_{1}^{X}(E)\right)$ and an event $e$ such that $\kappa \cup\{e\} \in$ $\operatorname{Configs}\left(\Phi_{1}^{X}(E)\right)$,

$$
\Phi_{2}(\kappa \cup\{e\})=\Phi_{2}(\kappa) / e
$$

\section{D.2 Lemmas}

Lemma 2 For traces $t_{1}, t_{2}$ and a single event action a

$$
\left(t_{1} \mid\{a\}\right)\left|t_{2}=t_{1}\right|\left(\{a\} \mid t_{2}\right)=\left(t_{1} \mid t_{2}\right) \mid\{a\}
$$

Proof : Follows directly from the definition of $\mid$ over traces.

Lemma 3 If trace $s=\mathcal{E}\left(e_{1}, \ldots e_{n}\right)$ and event e has all its causal predecessors in $\left\{e_{1}, \ldots e_{n}\right\}$, then for any trace $t$,

$$
(s \mid t) / e=(s / e) \mid t
$$

Proof : Follows directly from the definition of $\mid$ and /over traces.

Lemma 4 For a heap $E$ and $\kappa \in \operatorname{Configs}\left(\Phi_{1}^{X}(E)\right)$, if $e \in \Phi_{1}^{X}(E)-\kappa$ is such that it is concurrent to all events in $\kappa$ and $\kappa \cup\{e\} \in \operatorname{Configs}\left(\Phi_{1}^{X}(E)\right)$, then

$$
\Phi_{2}(\kappa \cup\{e\})=\Phi_{2}(\kappa) \mid \Phi_{2}(\{e\})
$$

Proof : Since $e$ is concurrent to all events in $\kappa$, the linearizations $\Phi_{2}(\kappa \cup\{e\})$ is all the possible interleavings of linearizations $\Phi_{2}(\kappa)$ and $\Phi_{2}(\{e\})$. 
Lemma 5 For two heaps $E, F$, a fixed set of variables $X$ and distinct marks $l, r$ :

$$
\Phi_{1}^{X}\left(E^{l} \cup F^{r}\right)=\Phi_{1}^{X}\left(E^{l}\right) \cup \Phi_{1}^{X}\left(F^{r}\right)
$$

Proof: Follows from the definition of $\Phi_{1}^{X}$. The common events in $\Phi_{1}^{X}\left(E^{l}\right)$ and $\Phi_{1}^{X}\left(F^{r}\right)$ are the events $e_{x}$ for all $x \in X$ which also belong to $\Phi_{1}^{X}\left(E^{l} \cup F^{r}\right)$. All the others events are distinct due to the marks $l$ and $r$ and so appear separately in $\Phi_{1}^{X}\left(E^{l} \cup F^{r}\right)$.

As a consequence of this lemma, we have that for any $K \subseteq \Phi_{1}^{X}\left(E^{l} \cup F^{r}\right)$ there exist unique maximal (w.r.t set inclusion) sets $K_{1} \subseteq \Phi_{1}^{X}\left(E^{l}\right)$ and $K_{2} \subseteq \Phi_{1}^{X}\left(F^{r}\right)$ such that $K=K_{1} \cup K_{2}$. Moreover if $K$ is a configuration of $\Phi_{1}^{X}\left(E^{l} \cup F^{r}\right)$, then $K_{1}$ and $K_{2}$ are configurations of $\Phi_{1}^{X}\left(E^{l}\right)$ and $\Phi_{1}^{X}\left(F^{r}\right)$ respectively, since they satisfy the conditions of Definition 2

Lemma 6 For $\kappa_{1} \in \operatorname{Configs}\left(\Phi_{1}^{X}\left(E^{l}\right)\right), \kappa_{2} \in \operatorname{Configs}\left(\Phi_{1}^{X}\left(F^{r}\right)\right)$

$$
\Phi_{2}\left(\kappa_{1} \cup \kappa_{2}\right)=\Phi_{2}\left(\kappa_{1}\right) \mid \Phi_{2}\left(\kappa_{2}\right)
$$

Proof: We prove this by induction on the size of the configuration. The base case is obvious when both configurations have a single event. If this event is common to both $\kappa_{1}$ and $\kappa_{2}$ (an event of the form $e_{x}$ ), both sides of the above equation is simply the trace consisting of this single event. If the events are distinct, both sides of the equation are the two traces in which the order of the events are interchanged.

Now suppose that the Lemma holds for configurations $\kappa_{1} \in \operatorname{Configs}\left(\Phi_{1}^{X}\left(E^{l}\right)\right)$ and $\kappa_{2} \in$ $\operatorname{Configs}\left(\Phi_{1}^{X}\left(F^{r}\right)\right)$ i.e,

$$
\Phi_{2}\left(\kappa_{1} \cup \kappa_{2}\right)=\Phi_{2}\left(\kappa_{1}\right) \mid \Phi_{2}\left(\kappa_{2}\right)
$$

Consider the configuration $\kappa_{1} \cup\{e\} \in \operatorname{Configs}\left(\Phi_{1}^{X}\left(E^{l}\right)\right)$.

Case 1: $e$ is minimal in $\kappa_{1}$ i.e $\nexists e^{\prime} \in \kappa_{1}$ such that $e \prec e^{\prime}$. This also means that $e$ is concurrent to all events in $\kappa_{1}$ and is a configuration in itself. From Lemma 4

$$
\Phi_{2}\left(\kappa_{1} \cup\{e\}\right)=\Phi_{2}\left(\kappa_{1}\right) \mid \Phi_{2}(\{e\})
$$

There are two possibilities here. If $e \in \kappa_{2}$, then $e$ is a substitution event of the kind $e_{x}$. Here $\Phi_{2}\left(\kappa_{1} \cup\{e\} \cup \kappa_{2}\right)=\Phi_{2}\left(\kappa_{1} \cup \kappa_{2}\right)=\Phi_{2}\left(\kappa_{1}\right) \mid \Phi_{2}\left(\kappa_{2}\right)$ by the hypothesis. Also

$$
\begin{aligned}
\Phi_{2}\left(\kappa_{1} \cup\{e\}\right) \mid \Phi_{2}\left(\kappa_{2}\right) & =\left(\Phi_{2}\left(\kappa_{1}\right) \mid \Phi_{2}(\{e\})\right) \mid \Phi_{2}\left(\kappa_{2}\right) \quad(\text { Lemma 4) } \\
& =\Phi_{2}\left(\kappa_{1}\right) \mid\left(\Phi_{2}(\{e\}) \mid \Phi_{2}\left(\kappa_{2}\right)\right) \quad(\text { Lemma 2) } \\
& =\Phi_{2}\left(\kappa_{1}\right) \mid \Phi_{2}\left(\kappa_{2}\right) \quad\left(\text { since } e \text { appears in } \kappa_{2}\right) \\
& =\Phi_{2}\left(\kappa_{1} \cup\{e\} \cup \kappa_{2}\right)
\end{aligned}
$$

If $e \notin \kappa_{2}$, then $e$ is concurrent to both $\kappa_{1}$ and $\kappa_{2}$. Hence

$$
\begin{aligned}
\Phi_{2}\left(\kappa_{1} \cup\{e\} \cup \kappa_{2}\right) & =\Phi_{2}\left(\kappa_{1} \cup \kappa_{2}\right) \mid \Phi_{2}(\{e\}) \quad(\text { Lemma 4) } \\
& =\left(\Phi_{2}\left(\kappa_{1}\right) \mid \Phi_{2}\left(\kappa_{2}\right)\right) \mid \Phi_{2}(\{e\}) \\
& =\left(\Phi_{2}\left(\kappa_{1}\right) \mid \Phi_{2}(\{e\})\right) \mid \Phi_{2}\left(\kappa_{2}\right) \quad(\text { Lemma 2) } \\
& =\Phi_{2}\left(\kappa_{1} \cup\{e\}\right) \mid \Phi_{2}\left(\kappa_{2}\right) \quad(\text { Lemma 4) }
\end{aligned}
$$


Case 2: $\quad e$ is not minimal in $\kappa_{1}$. Since all the causal predecessors of $e$ are in $\kappa_{1}, \Phi_{2}\left(\kappa_{1} \cup\right.$ $\{e\})=\Phi_{2}\left(\kappa_{1}\right) / e$. Also,

$$
\begin{aligned}
\Phi_{2}\left(\kappa_{1} \cup\{e\} \cup \kappa_{2}\right) & =\Phi_{2}\left(\kappa_{1} \cup \kappa_{2}\right) / e \\
& =\left(\Phi_{2}\left(\kappa_{1}\right) \mid \Phi_{2}\left(\kappa_{2}\right)\right) / e \quad \text { (Hypothesis) } \\
& =\left(\Phi_{2}\left(\kappa_{1}\right) / e \mid \Phi_{2}\left(\kappa_{2}\right)\right) \quad \text { (Lemma 3) } \\
& =\Phi_{2}\left(\kappa_{1} \cup\{e\}\right) \mid \Phi_{2}\left(\kappa_{2}\right)
\end{aligned}
$$

Lemma 7 For heaps $E, F$ and a set of variables $X \supseteq X_{E} \cup X_{F}$

$$
\Phi_{3}^{X}\left(E^{l} \cup F^{r}\right)=\Phi_{3}^{X}\left(E^{l}\right) \mid \Phi_{3}^{X}\left(F^{r}\right)
$$

Proof:

$$
\begin{aligned}
& \Phi_{3}^{X}\left(E^{l} \cup F^{r}\right)=\bigcup_{\kappa \in \operatorname{Configs}\left(\Phi_{1}^{X}\left(E^{l} \cup F^{r}\right)\right)} \Phi_{2}(\kappa) \\
& =\bigcup_{\kappa_{1} \in \operatorname{Configs}\left(\Phi_{1}^{X}\left(E^{l}\right)\right)} \Phi_{2}\left(\kappa_{1} \cup \kappa_{2}\right) \quad \text { (Lemma 5) } \\
& \kappa_{2} \in \operatorname{Configs}\left(\Phi_{1}^{X}\left(F^{r}\right)\right) \\
& =\bigcup_{\kappa_{1} \in \operatorname{Configs}\left(\Phi_{1}^{X}\left(E^{l}\right)\right)}\left(\Phi_{2}\left(\kappa_{1}\right) \mid \Phi_{2}\left(\kappa_{2}\right)\right) \quad(\text { Lemma 6) } \\
& \kappa_{2} \in \operatorname{Configs}\left(\Phi_{1}^{X}\left(F^{r}\right)\right) \\
& =\left(\bigcup_{\kappa_{1} \in \operatorname{Configs}\left(\Phi_{1}^{X}\left(E^{l}\right)\right)} \Phi_{2}\left(\kappa_{1}\right)\right) \mid\left(\bigcup_{\kappa_{2} \in \operatorname{Configs}\left(\Phi_{1}^{X}\left(F^{r}\right)\right)} \Phi_{2}\left(\kappa_{2}\right)\right) \\
& =\Phi_{3}^{X}\left(E^{l}\right) \mid \Phi_{3}^{X}\left(F^{r}\right)
\end{aligned}
$$

\section{Lemma 8}

$$
\bigcup_{X \supseteq X_{E \uplus F}}\left(\Phi_{3}^{X}(E) \mid \Phi_{3}^{X}(F)\right)=\bigcup_{X \supseteq X_{E}} \Phi_{3}^{X}(E) \mid \bigcup_{X \supseteq X_{F}} \Phi_{3}^{X}(F)
$$

Proof: The term on the left is clearly contained in the term on the right side of the equation. Now for any $t_{1} \in \Phi_{3}^{X_{1}}(E)$ and $t_{2} \in \Phi_{3}^{X_{2}}(F)$, the trace $t_{1} \mid t_{2}$ in the right term merges the common substitutions events for variables in $X_{1}$ and $X_{2}$. It also includes the substitution events that are not common to $X_{1}$ and $X_{2} . t_{1} \mid t_{2}$ is thus contained in the left side term when we take $X=X_{1} \cup X_{2}$.

Lemma 9 If trace $t_{1}[v / x] t_{2} \in \Phi(E)$ for a heap $E$, then $t_{1} t_{2} \in \Phi([v / x]$.E). Where heap $[v / x] . E$ is obtained by replacing variable $x$ by $v$ in the action of all events in $E$. 


\section{D.3 Correspondence Theorem}

For all Orc expressions $f$,

$$
\langle f\rangle=\Phi([f])
$$

Proof: The proof is by induction on the structure of $f$. The set of traces $\langle f\rangle$ differ from those defined in [7] in that we only consider traces where for any variable $x$, there is at most one substitution event $[v / x]$. This restriction is justified since once a variable $x$ has been substituted by a value $v$, future substitutions for $x$ leave the expression unchanged.

D.3.1 $\bullet f=\operatorname{let}(v)$

$v$ is a constant: By definition, $\langle l e t(v)\rangle=\{! v\}$. Since $[\operatorname{let}(v)]$ has only one event with the label $! v, \Phi([\operatorname{let}(v)])$ is $\{! v\}$.

$v$ is a variable $x$ : If we consider only the maximal traces $\langle$ let $(x)\rangle=\{[v / x], ! v\}$ (Other traces are just prefixes of the maximal traces). Since the only event $e$ in $[$ let $(x)]$ has $x$ as a free variable, $\Phi_{1}^{X}([\operatorname{let}(x)])$ by construction has an event $e_{x}$ causally preceding $e .\left\{e_{x}, e\right\}$ is the only maximal configuration of $\Phi_{1}^{X}([\operatorname{let}(x)])$ and $\Phi_{2}\left(\left\{e_{x}, e\right\}\right)=\{[v / x], ! v\}$. Thus $\Phi([\operatorname{let}(x)])=$ $\langle\operatorname{let}(x)\rangle$.

D.3.2 $\bullet f=? k$.

Considering only maximal traces, $\langle ? k\rangle=\{k ? v, ! v\}$. From section 4.1$][k]=\left\{e_{1}, e_{2}\right\}$ where $e_{1} \prec e_{2}$ and $\alpha\left(e_{1}\right)=k ? v, \alpha\left(e_{2}\right)=! v$. Clearly $\left\{e_{1}, e_{2}\right\}$ is the only maximal configuration of $[? k]$ and thus $\Phi([? k])=\{k ? v, ! v\}$.

D.3.3 $\bullet=M(v)$.

$v$ is a constant: Similar to the proof for ?k, $\langle M(v)\rangle=\left\{M_{k}(v), k ? v_{k}, ! v_{k}\right\}$ and $[M(v)]=$ $\left\{e_{1}, e_{2}, e_{3}\right\}$ where $e_{1} \prec e_{2} \prec e_{3}$ and $\alpha\left(e_{1}\right)=M_{k}(v), \alpha\left(e_{2}\right)=k ? v_{k}, \alpha\left(e_{3}\right)=! v_{k}$.

$v$ is a variable $x^{\prime}$ : Here $\langle M(x)\rangle=\{[v / x]\langle M(v)\rangle\}$ Now $[M(x)]=\left\{e_{1}, e_{2}, e_{3}\right\}$ where $e_{1} \prec e_{2} \prec e_{3}$ and $\alpha\left(e_{1}\right)=M_{k}(x), \alpha\left(e_{2}\right)=k ? v_{k}, \alpha\left(e_{3}\right)=! v_{k}$. Similar to the let $(x)$ case, $\Phi_{1}^{X}\left([M(x)]\right.$ would have the additional event $e_{x}$ causally preceding $e_{1}$. Thus $e_{x} \prec e_{1} \prec e_{2} \prec$ $e_{3}$ and $\left\{e_{x}, e_{1}, e_{2}, e_{3}\right\}$ is the only maximal configuration of $\Phi_{1}^{X}\left([M(x)] . \Phi_{2}\left(\left\{e_{x}, e_{1}, e_{2}, e_{3}\right\}\right)\right.$ is $\left\{[v / x], M_{k}(v), k ? v_{k}, ! v_{k}\right\}=\langle M(x)\rangle$.

D.3.4 $\bullet f \mid g$.

Here we need to prove that $\langle f \mid g\rangle=\Phi([f \mid g])$. We have

$$
\langle f \mid g\rangle=\langle f\rangle|\langle g\rangle=\Phi([f])| \Phi([g])
$$


from the recursive hypothesis. We thus need to show that

$$
\Phi([f \mid g])=\Phi([f]) \mid \Phi([g])
$$

Now,

$$
\begin{aligned}
& \Phi([f \mid g])=\bigcup_{X \supseteq X_{[f \mid g]}} \Phi_{3}^{X}([f \mid g]) \\
& =\bigcup_{X \supseteq X_{[f \mid g]}} \Phi_{3}^{X}\left([f]^{l e f t} \cup[g]^{\text {right }}\right) \\
& =\bigcup_{X \supseteq X_{[f \mid g]}}\left(\Phi_{3}^{X}\left([f]^{l e f t}\right) \mid \Phi_{3}^{X}\left([g]^{\text {right }}\right)\right) \text { (Lemma (7) } \\
& =\bigcup_{X \supseteq X_{[f] \uplus[g]}}\left(\Phi_{3}^{X}([f]) \mid \Phi_{3}^{X}([g])\right) \\
& =\bigcup_{X \supseteq X_{[f]}} \Phi_{3}^{X}([f]) \mid \bigcup_{X \supseteq X_{[g]}} \Phi_{3}^{X}([g]) \quad \text { (Lemma 8) } \\
& =\Phi([f]) \mid \Phi([g])
\end{aligned}
$$

\section{D.3.5 $\bullet f>x>g$}

We need to show that $\langle f>x>g\rangle=\Phi([f>x>g])$. We have

$$
\langle f>x>g\rangle=\langle f\rangle>x>\langle g\rangle=\Phi([f])>x>\Phi([g])
$$

so it is enough to show that

$$
\Phi([f])>x>\Phi([g])=\Phi([f>x>g])
$$

We successively prove $\subseteq($ Part 1$)$ and then $\supseteq$ (Part 2). By Definition,

$$
[f>x>g]=\operatorname{SEND}([f]) \cup \bigcup_{e \in ![f], \alpha(e)=! v} \operatorname{LiNK}(\tau(e), v, x,[g], \emptyset)
$$

Part 1. $\Phi([f])>x>\Phi([g]) \subseteq \Phi([f>x>g])$

$$
\Phi([f])>x>\Phi([g])=\{s>x>\Phi([g]) \mid s \in \Phi([f])\}
$$

We prove that for any $s \in \Phi([f]), s>x>\Phi([g]) \in \Phi([f>x>g])$. We do this by induction on the number of publication events in $s$.

$s$ has no publication events : This means that the configuration $\kappa \in \operatorname{Configs}\left(\Phi_{1}^{X}([f])\right)$ for which $s \in \Phi_{2}(\kappa)$ holds has no publication event either. So $\operatorname{SEND}(\kappa)=\kappa$ is a configuration of $\Phi_{1}^{X}(\operatorname{SEnd}([f]))$. Since $\operatorname{SEnd}([f]) \subseteq[f>x>g]$ (from (25]) $), \kappa \in \operatorname{Configs}\left(\Phi_{1}^{X}([f>x>g])\right)$. Thus $s \in \Phi([f>x>g])$. 
$s=r ! v t, r$ has no publications: By Definition,

$$
s>x>\Phi([g])=r(t>x>D . \Phi([g]) \mid D \cdot[v / x] \Phi([g]))
$$

where $D$ is the sequence of substitutions in $r$. Let $e$ be the publication event of $[f]$ in the trace $s$ with $\alpha(e)=! v$ and let $f \stackrel{r}{\Rightarrow} f^{\prime \prime} \stackrel{! v}{\longrightarrow} f^{\prime} \stackrel{t}{\Rightarrow}$.

Let $\kappa_{r} \in \mathbf{C o n f i g s}\left(\Phi_{1}^{X}([f])\right)$ be the configuration behind the trace $r$. Since $\kappa_{r} \cup\{e\}$ has only one publication event $e, r \cdot \tau=r \in \Phi_{2}\left(\kappa_{r}^{\prime} \cup\{\tau(e)\}\right)$ where $\kappa_{r}^{\prime} \cup\{\tau(e)\}$ is a configuration of $\Phi_{1}^{X}(\operatorname{SEND}([f]))$. Similarly, for $\kappa_{t}$ the configuration for the trace $t$ in $\left[f^{\prime}\right]$, the set of corresponding events $\kappa_{t}^{\prime}$ is a configuration of $\Phi_{1}^{X}\left(\operatorname{SEND}\left(\left[f^{\prime}\right]\right)\right)$. Since $\kappa_{r} \cup\{e\} \cup \kappa_{t}$ is a configuration of $\Phi_{1}^{X}([f])$,

$$
\kappa_{r}^{\prime} \cup\{\tau(e)\} \cup \kappa_{t}^{\prime} \in \operatorname{Configs}\left(\Phi_{1}^{X}(\operatorname{SEnD}([f]))\right)
$$

Now $f^{\prime} \stackrel{t}{\Rightarrow}$. Since $t$ has one less publication than $s$, by the initial hypothesis we have

$$
t>x>D . \Phi([g])=t>x>\Phi([D . g]) \in \Phi\left(f^{\prime}>x>D . g\right)
$$

i.e any trace $p \in t>x>D . \Phi([g])$ is such that $p \in \Phi_{2}\left(\kappa_{p}\right)$ where

$$
\kappa_{p} \in \operatorname{Configs}\left(\Phi_{1}^{X}\left(\operatorname{SEnd}\left(\left[f^{\prime}\right]\right) \cup \bigcup_{e^{\prime} \in !\left[f^{\prime}\right], \alpha\left(e^{\prime}\right)=! v} \operatorname{Link}\left(\tau\left(e^{\prime}\right), v, x,[D . g], \emptyset\right)\right)\right)
$$

Since $\kappa_{t}^{\prime}$ is the configuration in $\operatorname{SEND}\left(\left[f^{\prime}\right]\right.$ that generates trace $t$,

$$
\kappa_{p} \in \operatorname{Configs}\left(\Phi_{1}^{X}\left(\kappa_{t}^{\prime} \cup \underset{e^{\prime} \in ![f] \backslash e, \alpha\left(e^{\prime}\right)=! v}{ } \operatorname{LINK}\left(\tau\left(e^{\prime}\right), v, x,[D . g], \emptyset\right)\right)\right)
$$

Combining (26) and (27) and observing that the sequence of substitution events $D$ in in $D .[g]$ is the same as in $\kappa_{r}^{\prime}$, we have

$$
\left.\left.\kappa_{r}^{\prime} \cup\{\tau(e)\} \cup \kappa_{p} \in \underset{e^{\prime} \in ![f] \backslash e, \alpha\left(e^{\prime}\right)=! v}{\operatorname{Configs}\left(\Phi_{1}^{X}(\operatorname{SEnD}([f]) \cup\right.} \operatorname{LinK}\left(\tau\left(e^{\prime}\right), v, x,[g], \emptyset\right)\right)\right)
$$

and that trace $r . \tau \cdot p=r p \in \Phi_{2}\left(\kappa_{r}^{\prime} \cup\{\tau(e)\} \cup \kappa_{p}\right)$.

Now, consider the heap $\operatorname{Link}(\tau(e), v, x,[g], \emptyset)$. Since the context heap is empty, this heap is just a copy of $[g]$, with variable $x$ replaced by $v$ and all minimal events having $\tau(e)$ as their causal predecessor. Hence for a trace $q \in D \cdot[v / x] . \Phi([g])$, for the corresponding set of events $\kappa_{q}$ in $\operatorname{LINK}(\tau(e), v, x,[g], \emptyset)$ is such that $D . q \in \Phi_{2}\left(\kappa_{q}\right) . \kappa_{q}$ is not a configuration since its minimal events have the external condition $\tau(e)$ as a predecessor, but $\kappa_{r}^{\prime} \cup\{\tau(e)\} \cup \kappa_{q}$ is a configuration of $\operatorname{SEND}(\kappa \cup\{e\}) \cup \operatorname{LinK}(\tau(e), v, x,[g], \emptyset)$. Moreover since the same substitution events ' $D$ ' in D.q occur in $r, r q \in \Phi_{2}\left(\kappa_{r}^{\prime} \cup\{\tau(e)\} \cup \kappa_{q}\right)$. 
Finally, it is easy to see that since $\kappa_{r}^{\prime} \cup\{\tau(e)\} \cup \kappa_{q}$ and $\kappa_{r}^{\prime} \cup\{\tau(e)\} \cup \kappa_{p}$ are both configurations, and the events in $\kappa_{p}$ and $\kappa_{q}$ are concurrent, $\kappa=\kappa_{r}^{\prime} \cup\{\tau(e)\} \cup \kappa_{p} \cup \kappa_{q}$ is a configuration of such that $r(p \mid q) \in \Phi_{2}(\kappa)$ where

$$
\begin{gathered}
\kappa \in \operatorname{Configs}\left(\Phi_{1}^{X}\left(\operatorname{SEnd}([f]) \cup \bigcup_{e^{\prime} \in ![f] \backslash e, \alpha\left(e^{\prime}\right)=! v} \operatorname{LinK}\left(\tau\left(e^{\prime}\right), v, x,[g], \emptyset\right)\right)\right. \\
\cup \operatorname{LinK}(\tau(e), v, x,[g], \emptyset))
\end{gathered}
$$

$$
\kappa \in \operatorname{Configs}\left(\Phi_{1}^{X}\left(\operatorname{SEnd}([f]) \cup \bigcup_{e \in ![f] \alpha(e)=! v} \operatorname{LINK}(\tau(e), v, x,[g], \emptyset)\right)\right.
$$

Hence $r(p \mid q) \in \Phi([f>x>g])$.

Part 2. $\Phi([f])>x>\Phi([g]) \supseteq \Phi([f>x>g])$

From (25), we see that all minimal events in the LINK heaps are preceded by a $\tau(e)$ event. Hence any configuration of $\kappa$ of $\Phi_{1}^{X}([f>x>g])$ necessarily has events from $\operatorname{SEnD}([f])$. We do the proof by induction on the number of such $\tau(e)$ events of $\operatorname{SEND}([f])$ in $\kappa$.

$\kappa$ has no $\tau(e)$ events in $\operatorname{SEND}([f])$ : This means that all events in $\kappa$ belong to $\Phi_{1}^{X}(\operatorname{SEND}([f]))$. Clearly, the set of events corresponding to $\kappa$ in $\Phi_{1}^{X}([f])$ is a configuration, with no publication events. Thus for any trace $s \in \Phi_{2}(\kappa), s \in \Phi_{2}\left(\kappa^{\prime}\right)$ where $\kappa^{\prime} \in \Phi_{1}^{X}([f])$. Thus $s \in \Phi([f])$. Since $s$ has no publish events, $s>x>\Phi([g])=s$, thus $s \in \Phi([f])>x>\Phi([g])$.

$\kappa$ has a $\tau(e)$ event in $\operatorname{SEND}([f])$ : Let $\kappa_{s}^{\prime}=\kappa \cap \Phi_{1}^{X}(\operatorname{SEND}([f]))$ be the events from $\left.\operatorname{SEND}([f])\right)$. Let $p \in \Phi_{2}(\kappa)$ be a trace of $\Phi([f>x>g])$. The restriction of trace $p$ to events in $\Phi_{1}^{X}(\operatorname{SEND}([f]))$ is $\kappa_{s}^{\prime}$. Now, the events corresponding to $\kappa_{s}^{\prime}$ in $[f]$ is a configuration of $\Phi_{1}^{X}([f])$. Consider the trace $s$ of this configuration which corresponds to $p$ ( $s$ is obtained by replacing all the $\tau(e)$ events in $p$ by $e$ ). Clearly $s$ is a trace of $[f]$. Let it be of the form $s=r ! v t$, where $r$ has no publication event and $D$ is the sequence of substitutions in $r$. Let $f \stackrel{r}{\Rightarrow} f^{\prime \prime} \stackrel{! v}{\longrightarrow} f^{\prime} \stackrel{t}{\Rightarrow}$.

Since none of the LINK heap events can occur till a $\tau(e)$ event occurs, trace $p$ starts with the trace $r$. The next event in $s$ is a publication event $e$, which corresponds to a $\tau(e)$ event in $p$. So $p$ can now have events from the heap $\operatorname{Link}(\tau(e), v, x, D .[g], \emptyset))$. Let $\kappa_{q}^{\prime}$ be the set of events in $p$ from this heap. Any linearization of $\kappa_{q} q$, is a trace in $D \cdot[v / x] \Phi([g])$.

The other possible events in $p, \kappa_{u}^{\prime}$ are the events in $\operatorname{SEND}([f])$ corresponding to the trace $t$ in $[f]$, and events from the corresponding LINK heaps for the $\tau(e)$ events in $t$. Since $f^{\prime} \stackrel{t}{\Rightarrow}$, and $t$ has one less $\tau(e)$ event than $s$, applying the recursive hypothesis we have that for any linearization $u$ of $\kappa_{u}^{\prime}, u \in t>x>D . \Phi([g])$.

Finally, we observe that events in $\kappa_{u}^{\prime}$ and $\kappa_{q}^{\prime}$ are concurrent to each other, and so their possible linearisations are given by $u \mid q$, for all linearisations $u, q$ of $\kappa_{u}^{\prime}$ and $\kappa_{q}^{\prime}$. Therefore the trace $p$ is of the form $r(u \mid q)$ where $u \in t>x>D . \Phi([g])$ and $q \in D \cdot[v / x] \Phi([g])$. 
D.3.6 $-f$ where $x: \in g$.

We need to show that $\langle f$ where $x: \in g\rangle=\Phi([f$ where $x: \in g])$, i.e to show that

$$
\Phi([f]) \text { where } x: \in \Phi([g])=\Phi([f \text { where } x: \in g])
$$

Part 1. $\Phi([f])$ where $x: \in \Phi([g]) \subseteq \Phi([f$ where $x: \in g])$ : Let $t_{1} \in \Phi([f])$ and $t_{2} \in \Phi([g])$. We show that $\left(t_{1}\right.$ where $\left.x: \in t_{2}\right)$ when defined, belongs to $\Phi([f$ where $x: \in g])$. Let $t_{1} \in \Phi_{2}\left(\kappa_{1}\right)$ where $\kappa_{1} \in \operatorname{Configs}\left(\Phi_{1}^{X_{1}}([f])\right)$ for some $X_{1}$ and $t_{2} \in \Phi_{2}\left(\kappa_{2}\right)$ where $\kappa_{2} \in$ $\operatorname{Configs}\left(\Phi_{1}^{X_{2}}([g])\right)$ for some $X_{2}$.

Case 1 : $t_{2}$ has no publication. This means that configuration $\kappa_{2}$ also has no publication event. Let $\kappa_{2}^{\prime}=\operatorname{SEnd}\left(\operatorname{STOP}\left(\kappa_{2}\right)\right)$. Then, $\kappa_{2}^{\prime} \in \operatorname{Configs}\left(\Phi_{1}^{X_{2}}(\operatorname{SEnd}(\operatorname{STOP}([g])))\right)$ and $t_{2} \in \Phi_{2}\left(\kappa_{2}^{\prime}\right)$. Let trace $t_{1}=t_{1}^{\prime}[v / x] t_{1}^{\prime \prime}$ where $t_{1}^{\prime}$ has no substitution on $x$. Clearly the events in $t_{1}^{\prime}$ do not depend on $x$ and so $t_{1}^{\prime} \in \Phi_{2}\left(\kappa_{1}^{\prime}\right)$ where $\kappa_{1}^{\prime} \in \operatorname{Configs}\left(\Phi_{1}^{X_{1}}([[f](\bar{x})])\right)$. By definition we have

$$
\begin{aligned}
& \left(t_{1} \text { where } x: \in t_{2}\right)=t_{1}^{\prime} \mid t_{2} \\
& \in \Phi_{2}\left(\kappa_{1}^{\prime}\right) \mid \Phi_{2}\left(\kappa_{2}^{\prime}\right) \\
& \in \Phi_{2}\left(\kappa_{1}^{\prime} \cup \kappa_{2}^{\prime}\right) \quad(\text { Lemma 6) } \\
& \in \Phi_{2}\left(\kappa^{\prime}\right) \quad \text { where } \kappa^{\prime} \in \operatorname{Configs}\left(\Phi_{1}^{X}(\operatorname{SEnd}(\operatorname{Stop}([g])) \cup[[f](\bar{x})])\right) \\
& \text { and } X=X_{1} \cup X_{2} \text {. (Lemma 5) } \\
& \in \quad \Phi_{2}(\kappa) \quad \kappa \in \operatorname{Configs}\left(\Phi_{1}^{X}(\operatorname{SEnd}(\operatorname{STOP}(G)) \cup(F(\bar{x})))\right) \\
& \text { and } F=[f]^{l e f t}, G=[g]^{\text {right }} \\
& \in \quad \Phi_{2}(\kappa) \quad \kappa \in \mathbf{C o n f i g s}\left(\Phi_{1}^{X}(\operatorname{SEnd}(\operatorname{Stop}(G)) \cup(F(\bar{x}))\right. \\
& \left.\left.\cup \operatorname{RECV}_{x}(\operatorname{STOP}(G), F(x), F(\bar{x}))\right)\right) \\
& \in \quad \Phi_{2}(\kappa) \quad \kappa \in \operatorname{Configs}\left(\Phi_{1}^{X}([f \text { where } x: \in g])\right. \\
& \in \Phi([f \text { where } x: \in g])
\end{aligned}
$$

Case $2: t_{2}$ has a publication. Let $t_{2}=t_{2}^{\prime} ! v t_{2}^{\prime \prime}, t_{2} \in \kappa_{2}$. Since the subset of $\kappa_{2}$ which generates $t_{2}^{\prime} ! v$ has only one publish event, $\operatorname{STOP}\left(\kappa_{2}\right) \in \operatorname{Configs}\left(\Phi_{1}^{X_{2}}(\operatorname{STOP}[g])\right)$. Clearly $t_{2}^{\prime} ! v \in \Phi_{2}\left(\operatorname{STOP}\left(\kappa_{2}\right)\right)$ since the event actions remain unchanged. Since the SEND operator only renames publish events to $\tau, t_{2}^{\prime} \tau \in \Phi_{2}\left(\operatorname{SEND}\left(\operatorname{STOP}\left(\kappa_{2}\right)\right)\right)$. Also since the trace discards the $\tau$ actions, $t_{2}^{\prime} \in \Phi_{2}\left(\operatorname{SEnd}\left(\operatorname{STOP}\left(\kappa_{2}\right)\right)\right)$ and thus $t_{2}^{\prime} \in \operatorname{Configs}\left(\Phi_{1}^{X_{2}}(\operatorname{Send}(\operatorname{Stop}(G)))\right)$ where $G=[g]^{\text {right }}$. Let $e$ be the $\tau$ event which was previously the publication event in $\kappa_{2}$. 
Now if $t_{1}=t_{1}^{\prime}[v / x] t_{1}^{\prime \prime} \in \Phi([f])$, from Lemma 9] we have

$$
\begin{aligned}
t_{1}^{\prime} t_{1}^{\prime \prime} & \in \Phi([v / x] \cdot[f]) \\
& \in \Phi([v / x] \cdot F) \text { where } F=[f]]^{l e f t} \\
& \in \Phi([v / x] \cdot(F(\bar{x}) \cup F(x)) \\
& \in \Phi\left([v / x] \cdot\left(F(\bar{x}) \cup \operatorname{COPY}_{e}(F(x), F(\bar{x}))\right)\right) \\
& \in \Phi\left(F(\bar{x}) \cup[v / x] \cdot \operatorname{COPY}_{e}(F(x), F(\bar{x}))\right)
\end{aligned}
$$

Now $\operatorname{LinK}(e, v, x, F(x), F(\bar{x}))$ is obtained by adding the event $e$ to the cause of the minimal events in $[v / x]$. COPY $(F(x), F(\bar{x})))$. Any configuration $\kappa$ having these events will require event $e$ (and its causal predecessors) to be in $\kappa$. Since all the events in $\left.[v / x] \cdot \operatorname{COPY}_{e}(F(x), F(\bar{x}))\right)$ depend on $x$, they occur in the subtrace $t_{1}^{\prime \prime}$. Hence the trace $\left(t_{1}^{\prime} \mid t_{2}^{\prime}\right) t_{1}^{\prime \prime}$ would be a linearization of $\kappa$.

$$
\begin{aligned}
\left(t_{1} \text { where } x: \in t_{2}\right)= & \left(t_{1}^{\prime} \mid t_{2}^{\prime}\right) t_{1}^{\prime \prime} \\
\in & \Phi_{2}(\kappa) \text { where } \\
& \kappa \in \operatorname{Configs}\left(\Phi_{1}^{X}(F(\bar{x}) \cup \operatorname{LINK}(e, v, x, F(x), F(\bar{x})) \cup \operatorname{SEND}(\operatorname{STOP}(G)))\right. \\
\in & \Phi_{2}(\kappa) \text { where } \kappa \in \operatorname{Configs}\left(\Phi_{1}^{X}([f \text { where } x: \in g])\right. \\
\in & \Phi([f \text { where } x: \in g])
\end{aligned}
$$

Part 2. $\Phi([f])$ where $x: \in \Phi([g]) \supseteq \Phi([f$ where $x: \in g])$ :

$$
[f \text { where } x: \in g]=\operatorname{SEnd}(\operatorname{STOP}(G)) \cup \operatorname{RECV}_{x}(\operatorname{STOP}(G), F(x), F(\bar{x})) \cup F(\bar{x})
$$

where $F=[f]^{\text {left }}$ and $G=[g]^{\text {right }}$. Any two publication events $e_{1}, e_{2}$ in $\operatorname{sTOP}(G)-$ and thus the corresponding $\tau$ events $\tau\left(e_{1}\right), \tau\left(e_{2}\right)$ in $\operatorname{SEND}(\operatorname{STOP}(G))$ - are mutually in conflict, and so they can not appear in the same configuration. Thus any $\kappa \in \operatorname{Configs}\left(\Phi_{1}^{X}([f\right.$ where $\left.x: \in g])\right)$ will have at most one such event $\tau(e)$ in it. Also, since each of the $\operatorname{LINK}(\tau(e), v, x, F(x), F(\bar{x}))$ heaps in $\operatorname{RECV}_{x}(\operatorname{STOP}(G), F(x), F(\bar{x}))$ has $\tau(e)$ in the causal preset of its minimal events, it follows that $\kappa$ cannot have events from two such LINK heaps since they would be in conflict.

Case $1: \kappa \cap \operatorname{send}(\operatorname{stop}(G))$ has no such $\tau(e)$ event. This means that the corresponding configuration in $G$ has no publication event $e$. Clearly $\kappa$ does not have events from $\operatorname{LINK}(\tau(e), v, x, F(x), F(\bar{x}))$ since they need $\tau(e)$ to appear in $\kappa$. Therefore $\kappa \in \operatorname{Configs}\left(\Phi_{1}^{X}(F(\bar{x}) \cup\right.$ $\operatorname{SEnd}(\operatorname{StOP}(G))))$. From Lemma $5=\kappa_{1} \cup \kappa_{2}$ where $\kappa_{1}$ and $\kappa_{2}$ are configurations of 


$$
\begin{aligned}
& \Phi_{1}^{X}(F(\bar{x})) \text { and } \Phi_{1}^{X}(\operatorname{SEND}(\operatorname{STOP}(G))) \text { respectively. Any trace } \\
& t \in \Phi_{2}(\kappa), \kappa \in \operatorname{Configs}\left(\Phi_{1}^{X}(F(\bar{x}) \cup \operatorname{SEnd}(\operatorname{Stop}(G)))\right) \\
& \in \Phi_{2}\left(\kappa_{1} \cup \kappa_{2}\right), \kappa_{1} \in \operatorname{Configs}\left(\Phi_{1}^{X}(F(\bar{x})), \kappa_{2} \in \operatorname{Configs}\left(\Phi_{1}^{X}(\operatorname{Send}(\operatorname{stop}(G)))\right)\right. \\
& \in \Phi_{2}\left(\kappa_{1} \cup \kappa_{2}\right), \kappa_{1} \in \operatorname{Configs}\left(\Phi_{1}^{X}(F)\right), \kappa_{2} \in \operatorname{Configs}\left(\Phi_{1}^{X}(\operatorname{Send}(\operatorname{stop}(G)))\right) \\
& \in \Phi_{2}\left(\kappa_{1}\right) \mid \Phi_{2}\left(\kappa_{2}\right), \kappa_{1} \in \operatorname{Configs}\left(\Phi_{1}^{X}(F)\right), \kappa_{2} \in \operatorname{Configs}\left(\Phi_{1}^{X}(\operatorname{Send}(\operatorname{stop}(G)))\right) \\
& \text { (Lemma 6) } \\
& \in \Phi_{2}\left(\kappa_{1}\right) \mid \Phi_{2}\left(\kappa_{2}\right), \kappa_{1} \in \operatorname{Configs}\left(\Phi_{1}^{X}(F)\right), \kappa_{2} \in \operatorname{Configs}\left(\Phi_{1}^{X}(G)\right) \\
& \text { Since } \kappa_{2} \text { has no publish events, traces } \operatorname{SEND}\left(\operatorname{STOP}\left(\kappa_{2}\right)\right) \text { remain the same } \\
& \in \Phi_{2}\left(\kappa_{1}\right) \mid \Phi_{2}\left(\kappa_{2}\right), \kappa_{1} \in \operatorname{Configs}\left(\Phi_{1}^{X}([f])\right), \kappa_{2} \in \operatorname{Configs}\left(\Phi_{1}^{X}([g])\right)
\end{aligned}
$$

Thus $t=t_{1} \mid t_{2}$ where $t_{1} \in \Phi([f]), t_{2} \in \Phi([g])$. Since $t_{2}$ has no publication event, $t=$ $t_{1}$ where $x: \in t_{2}$

Case $2: \kappa_{1}=\kappa \cap \operatorname{send}(\operatorname{stop}(G))$ has a $\tau(e)$ event. $\quad e$ is a publish event in $\operatorname{STOP}(G)$ and so in $G$ too. Let $\alpha(e)=! v$. Let $p$ be any trace in $\Phi_{2}(\kappa)$ and $t$ be the trace of the restriction of $p$ to events in $\kappa_{1}$. Since $\kappa_{1}$ is a configuration of $\operatorname{SEND}(\operatorname{STOP}(G))$, the preemption event $\tau(e)$ is the maximal event in any such linearization $t$.

From Lemma 1 the events in $G$ corresponding to $\kappa_{1}, \kappa_{1}^{\prime}=\operatorname{STOP}^{-1}\left(\operatorname{SEND}^{-1}\left(\kappa_{1}\right)\right)$ is a configuration of $G$. Consider the sequence of events in $\kappa_{1}^{\prime}$ which corresponds to the trace $t$ in $\kappa_{1}$. Such a sequence will have only one publication event $e$ which is furthermore the maximal event of the sequence. Since the only event whose label changes in the $\operatorname{SEND}^{-1}\left(\mathrm{STOP}^{-1}\left(\kappa_{1}\right)\right)$ transformation is the publication event $e$, and because $\alpha(\tau(e))=\tau$, the trace of the sequence of $\kappa_{1}^{\prime}$ events is $t ! v$, where $\alpha(e)=! v$. Thus $t ! v \in \Phi(G)$. Since $G$ is simply $[g]^{\text {right }}$, we have $t ! v \in \Phi([g])$.

The other two components of the configuration in $\kappa$ are the events $\kappa_{s}$ from $F(\bar{x})$ and $\kappa_{s^{\prime}}$ from $\operatorname{LINK}(\tau(e), v, x, F(x), F(\bar{x}))$. Let the sequence of events of $\kappa_{s}$ in $p$ be $s$ and that of $\kappa_{s^{\prime}}$ be $s^{\prime}$. Consider the events in $\kappa_{s^{\prime}}$. Since they are a copy of events in $F(x)$ with respect to the context $F(\bar{x})$, they have exactly the same causal and conflict relations with events in $F(\bar{x})$ that events in $F(x)$ have. Thus the events $\kappa_{s^{\prime}}^{\prime}$ in $F(x)$ corresponding to $\kappa_{s^{\prime}}$ are such that $\kappa_{s} \cup \kappa_{s^{\prime}}^{\prime}$ is a configuration of $F(x) \cup F(\bar{x})$, i.e, $F$. The LINK heap also replaces all occurrences of the variable $x$ by $v$ in its events.

Consider the sequence of events corresponding to the trace $p$ which belong to $\kappa_{s} \cup \kappa_{s^{\prime}}$. Let it be $e_{1}, \ldots e_{k-1}, e_{k}, \ldots . . e_{n}$ where $e_{k}$ is the first event of the sequence that belongs to $\kappa_{s^{\prime}}$. Let the trace of the sequence $e_{1}, \ldots e_{k-1}$ be $r$ and that of $e_{k}, \ldots e_{n}$ be $r^{\prime}$. If $e_{1}^{\prime}, \ldots e_{k-1}^{\prime}, e_{k}^{\prime}, \ldots . e_{n}^{\prime}$ is the corresponding sequence of events in $F$ (note that $e_{k}^{\prime}$ and all other events corresponding to $\kappa_{s^{\prime}}$ will have $x$ as a free variable here). Now since $\Phi_{1}^{X}(F)$ would add the substitution event $e_{x}$ to the causes of all events depending on $x$, and because $e_{k}^{\prime}$ is the first event in the trace that depends on $x,\left(e_{1}^{\prime}, \ldots e_{k-1}^{\prime} \mid e_{x}\right) e_{k}^{\prime} \ldots e_{n}^{\prime}$ is a linearization of $\kappa_{2}$ where $\kappa_{2} \in \operatorname{Configs}\left(\Phi_{1}^{X}(F)\right)$. In particular, $e_{1}^{\prime}, \ldots e_{k-1}^{\prime}, e_{x}, e_{k}, \ldots e_{n}^{\prime}$ is a linearization whose trace (sequence of actions) obtained by the transformation $\mathcal{E}$ (see section [5 Step 2.) is $r[v / x] r^{\prime}$. Thus $r[v / x] r^{\prime} \in$ $\Phi(F)=\Phi([f])$. 
Finally, we note that in trace $p$, all that events in $\kappa_{1}$ occur before $e_{k}\left(e_{k}\right.$ is the first event from $\kappa_{s^{\prime}}$ which has $\tau(e)$ as its causal predecessor, and $\tau(e)$ is the maximal event from $\kappa_{1}$ in $p$ ). $\kappa_{1}$ events are however concurrent to events in $\kappa_{s}$ and thus to $e_{1}, \ldots e_{k-1}$. Hence such a trace $p$ belongs to $(r \mid t) r^{\prime}$, where $t ! v \in \Phi\left([g]\right.$ and $r[v / x] r^{\prime} \in \Phi([f])$. Thus $p \in \Phi([f])$ where $x: \in \Phi([g])$. 
